\title{
Nonlinear Modeling and Dynamic Simulation Using Bifurcation and Stability Analyses of Regenerative Chatter of Ball-End Milling Process
}

\author{
Jeehyun Jung, Chinh Ngo, Dowung Son, and Jongwon Seok \\ School of Mechanical Engineering, College of Engineering, Chung-Ang University, 84 HeukSeok-Ro, DongJak-Gu, \\ Seoul 156-756, Republic of Korea \\ Correspondence should be addressed to Jongwon Seok; seokj@cau.ac.kr
}

Received 10 November 2015; Revised 15 January 2016; Accepted 18 January 2016

Academic Editor: Gen Qi Xu

Copyright (C) 2016 Jeehyun Jung et al. This is an open access article distributed under the Creative Commons Attribution License, which permits unrestricted use, distribution, and reproduction in any medium, provided the original work is properly cited.

\begin{abstract}
A dynamic model for a ball-end milling process that includes the consideration of cutting force nonlinearities and regenerative chatter effects is presented. The nonlinear cutting force is approximated using a Fourier series and then expanded into a Taylor series up to the third order. A series of nonlinear analyses was performed to investigate the nonlinear dynamic behavior of a ball-end milling system, and the differences between the nonlinear analysis approach and its linear counterpart were examined. A bifurcation analysis of points near the critical equilibrium points was performed using the method of multiple scales (MMS) and the method of harmonic balance (MHB) to analyse the local chatter behaviors of the system. The bifurcation analysis was conducted at two subcritical Hopf bifurcation points. It was also found that a ball-end milling system with nonlinear cutting forces near its critical equilibrium points is conditionally stable. The analysis and simulation results were compared with experimental data reported in the literature, and the physical significance of the results is discussed.
\end{abstract}

\section{Introduction}

Ball-end milling is one of the most widely used cutting processes for the machining of free-form surfaces. However, prediction of the cutting forces and vibrational behavior involved in ball-end milling processes is complicated because of the geometric complexity associated with the ball part of the tool. A typical obstacle to overcome in achieving high performance in ball-end milling is the well-known chatter phenomenon (i.e., chatter vibrations), which is typically caused by the regeneration of the waviness of the workpiece surface due to the tool path under specific operating conditions. Because accurate prediction of chatter vibration is essential to maximize the productivity of ball-end milling, it has been a topic of industrial and academic interest in the manufacturing sector for many years. Furthermore, to determine the stability of ball-end milling systems, investigation of the cutting force effect is also required. Based on the relation between the cutting force and the uncut chip thickness in the ball-end milling process, two types of models are commonly used to predict the cutting forces: nonlinear models [1-4] and linear models [5-9]. Nonlinearity in the cutting force model is known to exist because of the size effect [10]. Therefore, the consideration of nonlinearity in a cutting force model is important and should not be ignored to obtain accurate prediction of the dynamic behavior of a milling system [10-14].

Chatter in ball-end milling was first studied by Altıntaş and Lee in [15]. They described a numerical method for predicting stability lobes in a ball-end milling system. They integrated the mechanics and dynamics of ball-end milling into a time-domain simulation model, which resulted in a time-consuming computation process. The prediction of stability lobes in ball-end milling has been accomplished for a cylindrical end milling process [16]. In addition, the dynamics of ball-end milling has been successfully modeled with consideration of the distribution of the chip thickness in both the radial and axial directions [16]. In this modeling approach, the dynamic cutting force terms are linearized for various chip loads using the energy averaging technique. The final stability model is reduced to a quadratic delay differential equation (DDE). Abrari and Elbestawi [17] developed another method to predict the cutting forces associated with 
ball-end milling, which are essentially the same as those associated with flat-end milling. The only difference is that in ball-end milling, the cutting coefficients are expressed as functions of the cutting position along the cutting edge, whereas in flat-end milling, they are constant values. Abrari et al. [18] applied the method used for stability analysis of flatend milling systems [16] to ball-end milling systems. Ozturk and Budak [19] extended the three-dimensional chatter stability model to a five-axis ball-end milling system by considering the effects of the lead and tilt angles on the dynamic model and obtained both single-frequency and multiplefrequency solutions in which the variation in the cutting force coefficients was smoothed using averaging techniques. A new method, referred to as the chatter-free calibration method, was subsequently proposed for use in calculating the cutting forces in ball-end milling [20] and the associated stability chart was obtained using the semidiscretization method.

One common factor in the stability analysis studies reported in literature is that the stability lobe diagram is obtained for the final results; no further consideration is given to the effect of the nonlinearity of the system on behavior. The stability lobe separates the parameter domains of the spindle speed and depth of cut into parts corresponding to the stable region (free of chatter) and the unstable region (chatter).

In the present study, a nonlinear model for a ball-end milling process that includes the consideration of cutting force nonlinearities and the regenerative chatter effect was developed and compared to a corresponding linear model. The differences between the two models were clearly identified and addressed. The cutting force was expanded into a Taylor series up to the third order and expressed in terms of the zeroth order of a Fourier series. The system considered in this study was thereby modeled as a set of nonlinear autonomous DDEs, which results in difficulty in determining the stability boundary because of the existence of infinite eigenroots. To overcome this difficulty, the stability is often obtained in the frequency domain [17-20]. In this study, bifurcation and stability analyses of regenerative chatter in the nonlinear model for the cutting force in the ball-end milling process were performed through a series of systematic approaches that included linear stability analysis and the methods of multiple scales and harmonic balance [21] (MMS and MHB, resp.). We considered only the nonlinearity originating from the cutting force, we ignored other nonlinear effects such as the nonlinear stiffness of the cutting tool (or workpiece), nonsmoothness due to contact loss between the tool and the workpiece, and nonlinear process damping. The MMS and MHB were used to investigate the local dynamics of the system near its equilibrium points. Modulation equations were derived for the amplitude and phase angle of the periodic solutions and serve as normal forms of the governing equations representing the nonlinear dynamics of the ballend milling system considered. The resulting bifurcation diagrams for the limit cycle solutions reveal that subcritical Hopf bifurcations can originate from the two points investigated in this study along the boundary curves. It was also found that a ball-end milling system with nonlinear cutting forces near the critical equilibrium points is conditionally stable. The analysis
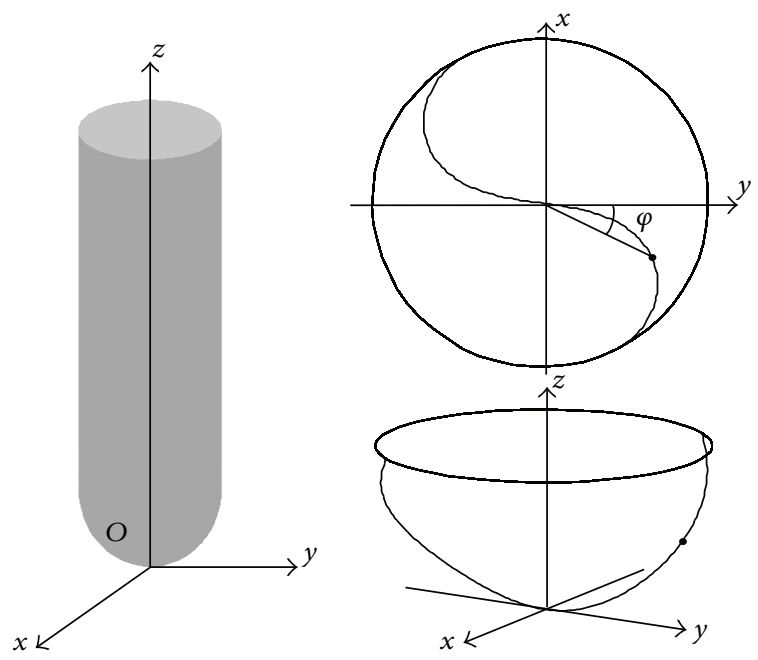

FIgURE 1: Ball-end milling tool geometry.

results were compared with experimental data reported in the literature, and their physical significance is discussed.

\section{Equations of Motion for Ball-End Milling System}

In a ball-end milling system, the angular position of a differential element on the $i$ th cutting edge (see Figure 1) can be obtained $[15,22]$ as follows:

$$
\varphi_{i}(z, t)=\frac{2 \pi \Omega}{60} t+(i-1) \frac{2 \pi}{N_{f}}-\frac{z}{R_{0}} \tan i_{0},
$$

where $\Omega, N_{f}, i_{0}$, and $R_{0}$ are the spindle speed, the number of teeth, the helix angle, and the radius of the cutter, respectively. If the feed rate is much smaller than the cutting speed, a circular tooth path can be used to approximate the tool path with a good accuracy [1]. The uncut chip thickness can be separated into the dynamic chip thickness $h_{i, \text { dyn }}\left(u_{i}\right)$ and static chip thickness $h_{i, \text { sta }}\left(s_{i}\right)$ as follows:

$$
\begin{aligned}
h_{i}= & h_{i, \mathrm{dyn}}+h_{i, \mathrm{sta}}, \\
h_{i, \mathrm{dyn}}= & u_{i} \\
= & (x(t)-x(t-\tau)) \sin \varphi_{i} \\
& +(y(t)-y(t-\tau)) \cos \varphi_{i}, \\
h_{i, \mathrm{sta}}= & s_{i}=f_{z} \sin \varphi_{i},
\end{aligned}
$$

where $\tau=60 /\left(N_{f} \Omega\right)$ is the time delay and $f_{z}$ is the feed per tooth of cutting process.

The relationship between the cutting force and the chip thickness that was employed in the present study is represented by as follows [1]:

$$
\begin{aligned}
& \mathrm{d} F_{T, i}\left(z, \varphi_{i}\right)=g\left(\varphi_{i, z}\right) K_{T}(z)\left[h_{i}\left(\varphi_{i}\right)\right]^{m_{T}} \mathrm{~d} z, \\
& \mathrm{~d} F_{R, i}\left(z, \varphi_{i}\right)=g\left(\varphi_{i, z}\right) K_{R}(z)\left[h_{i}\left(\varphi_{i}\right)\right]^{m_{R}} \mathrm{~d} z,
\end{aligned}
$$


where $\mathrm{d} F_{T, i}\left(z, \varphi_{i}\right)$ and $\mathrm{d} F_{R, i}\left(z, \varphi_{i}\right)$ are the differential tangential and radial cutting force components of a cutting element, respectively; $K_{T}(z)$ and $K_{R}(z)$ are the cutting-mechanics parameters for horizontal cutting motions, which play a role in characterizing the local cutting mechanics of the differential cutting edge. Unlike the cylindrical end, the ball end of a ball-end mill is considered in the present model through these cutting-mechanics parameters $\left(K_{T}\right.$ and $\left.K_{R}\right)$, which vary along the tool axis ( $z$-axis) and are generally determined from experimental data [1], and $m_{T}$ and $m_{R}$ are the exponents of the chip thickness, which explicitly characterize the size effect in metal cutting [4]. $g\left(\varphi_{i, z}\right)$ is a screen function that is equal to 1 if the $i$ th cutting edge is in the cut; otherwise, it is equal to 0 . Note that these exponents play a role in characterizing the size effect of the workpiece material and are typically assumed to be constants for a particular material. Also note that when the uncut chip thickness is less than 0 (i.e., $h_{i}<0$ ), $\mathrm{d} F_{T, i}=\mathrm{d} F_{R, i}=0$. This situation corresponds to loss of contact between the tool and the workpiece, which was not addressed in the present study.

The differential forces in the $x$ and $y$ directions can be obtained by coordinate transformation of the differential forces in the tangential and radial directions as follows:

$$
\left[\begin{array}{l}
\mathrm{d} F_{x, i} \\
\mathrm{~d} F_{y, i}
\end{array}\right]=\left[\begin{array}{cc}
-\cos \varphi_{i} & -\sin \varphi_{i} \\
\sin \varphi_{i} & -\cos \varphi_{i}
\end{array}\right]\left[\begin{array}{l}
\mathrm{d} F_{T, i} \\
\mathrm{~d} F_{R, i}
\end{array}\right] .
$$

To calculate the cutting forces accurately, it is convenient to divide the cutting tool into a finite number $(n)$ of thinly sliced disk elements along the $z$-axis. The cutting forces in the $x$ and $y$ directions on the $i$ th flute and $m$ th disk can then be individually calculated. By summing the individual cutting forces over the entire set of teeth, the total cutting forces acting on the $m$ th disk can be calculated. Finally, the total cutting forces acting on the tool can be obtained by summing the individual dynamic forces acting on each disk element over the entire domain of the tool, which results in the following expression:

$$
\begin{aligned}
& {\left[\begin{array}{l}
F_{x} \\
F_{y}
\end{array}\right]=\delta_{z} \sum_{m=1}^{n} \sum_{i=1}^{N} g\left(\varphi_{i, m}\right)} \\
& \cdot\left[\begin{array}{cc}
-\cos \varphi_{i, m} & -\sin \varphi_{i, m} \\
\sin \varphi_{i, m} & -\cos \varphi_{i, m}
\end{array}\right]\left[\begin{array}{l}
K_{T, m}\left[s_{i, m}+u_{i, m}\right]^{m_{T}} \\
K_{R, m}\left[s_{i, m}+u_{i, m}\right]^{m_{R}}
\end{array}\right],
\end{aligned}
$$

where $n$ is the number of disk elements and $\delta_{z}$ is the thickness of one disk.

The governing differential equations of the ball-end milling system can be expressed in their usual forms:

$$
\begin{aligned}
& m_{x} \ddot{x}+c_{x} \dot{x}+k_{x} x=F_{x}, \\
& m_{y} \ddot{y}+c_{y} \dot{y}+k_{y} x=F_{y}
\end{aligned}
$$

or

$$
\begin{aligned}
& \ddot{x}+2 \zeta_{x} \dot{x}+\omega_{x}^{2} x=\frac{1}{m_{x}} F_{x}, \\
& \ddot{y}+2 \zeta_{y} \dot{y}+\omega_{y}^{2} x=\frac{1}{m_{y}} F_{y},
\end{aligned}
$$

with

$$
\begin{aligned}
& \omega_{x}=\sqrt{\frac{k_{x}}{m_{x}}}, \\
& \omega_{y}=\sqrt{\frac{k_{y}}{m_{y}}}, \\
& \zeta_{x}=\frac{c_{x}}{2 m_{x}}, \\
& \zeta_{y}=\frac{c_{y}}{2 m_{y}},
\end{aligned}
$$

where $m, c, k$, and $\omega$ denote the modal mass, modal damping, modal stiffness, and natural angular frequency, respectively, and the subscripts $x$ and $y$ correspond to the $x$ and $y$ directions, respectively.

In (5) and (7), the proper ranges of several model parameters are known a priori, namely, $s_{i, m} \geq 0$ and $0<m_{T}, m_{R}<1$ for most metallic materials [1]. Note that the governing differential equations given above are actually nonlinear DDEs with a constant time delay. We assume that the displacements $(x, y)$ can be separated into their periodic and dynamic components according to the following equations:

$$
\begin{aligned}
& x(t)=x_{s}(t)+x_{p}(t), \\
& y(t)=y_{s}(t)+y_{p}(t),
\end{aligned}
$$

where $\left(x_{s}, y_{s}\right)$ is the steady-state periodic solution set of the displacements and $\left(x_{p}, y_{p}\right)$ is the dynamic perturbation solution set around $\left(x_{s}, y_{s}\right)$. In fact, $\left(x_{s}, y_{s}\right)$ is the solution for the unperturbed ideal case when no self-excited vibrations arise.

Because of the coupling of the force terms in (7) via the relation given by (5), the periodic solution set $\mathbf{q}_{s}(t)=$ $\left[\begin{array}{ll}x_{s}(t) & y_{s}(t)\end{array}\right]^{\mathrm{T}}$ can be represented by the following inhomogeneous vector equation:

$$
\ddot{\mathbf{q}}_{s}(t)+\zeta \dot{\mathbf{q}}_{s}(t)+\omega \mathbf{q}_{s}(t)=\mathbf{M}^{-1} \mathbf{F}_{s},
$$

where $\boldsymbol{\zeta}, \boldsymbol{\omega}, \mathbf{M}$, and $\mathbf{F}_{s}$ are known parameters, the detailed expressions of which are given in Appendix A.

From (5) and (7), the Taylor series expressions of the noninteger power functions $\left(m_{T}, m_{R}\right)$ in (5), expanded at the steady-state periodic solution $\mathbf{q}_{s}$, yield the following differential equations:

$$
\begin{aligned}
& \ddot{x}_{p}+2 \zeta_{x} \dot{x}_{p}+\omega_{x}^{2} x_{p}=\frac{1}{m_{x}} F_{d, x}, \\
& \ddot{y}_{p}+2 \zeta_{y} \dot{y}_{p}+\omega_{y}^{2} x_{p}=\frac{1}{m_{y}} F_{d, y},
\end{aligned}
$$

where

$$
\begin{aligned}
& {\left[\begin{array}{c}
F_{d, x} \\
F_{d, y}
\end{array}\right]=\delta_{z} \sum_{m=1}^{n} \sum_{i=1}^{N} g\left(\varphi_{i, m}\right)} \\
& \cdot\left[\begin{array}{cc}
-\cos \varphi_{i} & -\sin \varphi_{i} \\
\sin \varphi_{i} & -\cos \varphi_{i}
\end{array}\right]\left[\begin{array}{l}
a_{1} u_{i, m}+a_{2} u_{i, m}^{2}+a_{3} u_{i, m}^{3} \\
b_{1} u_{i, m}+b_{2} u_{i, m}^{2}+b_{3} u_{i, m}^{3}
\end{array}\right] .
\end{aligned}
$$


The Taylor series expressions of the nonlinear cutting forces and the detailed expressions of the coefficients $a_{k}$ and $b_{k}(k=$ $1, \ldots, 3)$ are given in Appendix A.

The resulting dynamic cutting forces expressed in (12) can be written in the following forms:

$$
\begin{aligned}
{\left[\begin{array}{c}
F_{d, x} \\
F_{d, y}
\end{array}\right] } & =\delta_{z} \sum_{m=1}^{n} \sum_{i=1}^{N}\left(g\left(\varphi_{i, m}\right) \mathbf{B}^{i, m}\left(\varphi_{i, m}\right)\right) \mathbf{N} \\
& =\delta_{z} \sum_{m=1}^{n}\left(\mathbf{B}^{m}\right) \mathbf{N}
\end{aligned}
$$

where $\mathbf{B}^{m}$ is the summation of the individual $\mathbf{B}^{i, m}$ (the detailed expression of which is given in Appendix A); namely,

$$
\begin{aligned}
& \mathbf{B}^{m}=\sum_{i=1}^{N} g_{i}\left(\varphi_{i, m}\right) \mathbf{B}^{i, m}\left(\varphi_{i, m}\right), \\
& \mathbf{N} \\
& =\left[\begin{array}{lllllllll}
\Delta_{x} & \Delta_{y} & \Delta_{x}^{2} & \Delta_{x}^{3} & \Delta_{y}^{2} & \Delta_{y}^{3} & \Delta_{x} \Delta_{y} & \Delta_{x}^{2} \Delta_{y} & \Delta_{x} \Delta_{y}^{2}
\end{array}\right]^{\mathrm{T}},
\end{aligned}
$$

where

$$
\begin{aligned}
& \Delta_{x}=x_{p}(t)-x_{p}(t-\tau), \\
& \Delta_{y}=y_{p}(t)-y_{p}(t-\tau) .
\end{aligned}
$$

Because the angle position is a function of time, (13) can be rewritten as follows:

$$
\left[\begin{array}{c}
F_{d, x} \\
F_{d, y}
\end{array}\right]=\delta_{z} \sum_{m=1}^{n}\left(\mathbf{B}^{m}(t)\right) \mathbf{N} .
$$

In (17), $\mathbf{B}^{m}(t)$ is a time-dependent periodic directional coefficient matrix that can be expand into a Fourier series expansion [19] as follows:

$$
\begin{aligned}
\mathbf{B}^{m}(t) & =\sum_{r=-\infty}^{+\infty} \mathbf{B}_{r}^{m} e^{i w \omega_{N} t}, \\
\mathbf{B}_{r}^{m} & =\frac{1}{T} \int_{0}^{T} \mathbf{B}^{m}(t) e^{-i r \omega_{N} t} \mathrm{~d} t,
\end{aligned}
$$

where $T$ and $\omega_{N}$ are the tooth passing period and tooth passing angular frequency, respectively, which can be calculated from the number of teeth and the spindle speed.

In a milling process, vibrations are known to occur primarily for two reasons $[23,24]$. First, the cutting forces are periodic with respect to the tooth passing frequency $\omega_{N}$, and they cause forced vibrations that are excluded from the stability analysis because they are proportional to the mean chip thickness. Second, experimental observations have shown that, in a dynamic milling system, in which dynamic displacements are related to dynamic milling forces, the amplitude of vibrations at the chatter frequency $\omega_{c}$ is much higher than that of the forced vibrations of the harmonics of the tooth passing frequency $\omega_{N}$. In accordance with these observations, two separate formulations are given in the following: the first is a multifrequency solution that considers the harmonic terms in $\left[\mathbf{B}^{m}(t)\right]$ and the second is a single-frequency solution that considers only the vibrations at the chatter frequency; that is, the harmonic terms are neglected, and only the average values in the Fourier expansion of $\left[\mathbf{B}^{m}(t)\right]$ are retained. When the radial immersion of the cutting process is not too small, the effect of harmonic terms is not significant $[19,20$, 25]. Because high immersion between the cutting tool and workpiece is considered in this study, only the single-frequency solution is sought, meaning the zeroth order in (18),

$$
\mathbf{B}_{0}^{m}=\frac{1}{T} \int_{0}^{T} \mathbf{B}^{m}(t) \mathrm{d} t
$$

or in the form represented in the angular domain [19],

$$
\mathbf{B}_{0}^{m}=\frac{1}{\varphi_{p}} \int_{0}^{\varphi_{p}} \mathbf{B}^{m}\left(\varphi_{i}\right) \mathrm{d} t
$$

where $\varphi_{p}$ is the angle between two successive teeth; $\varphi_{p}=$ $2 \pi / N$.

The substitution of $\mathbf{B}_{0}^{m}$ into (17) yields the following:

$$
\left[\begin{array}{c}
F_{d, x} \\
F_{d, y}
\end{array}\right]=\delta_{z} \sum_{m=1}^{n}\left(\mathbf{B}_{0}^{m}\right) \mathbf{N} .
$$

When the number of axial disks $n$ is given, (21) can be represented as follows:

$$
\left[\begin{array}{c}
F_{d, x} \\
F_{d, y}
\end{array}\right]=\delta_{z} \widehat{\mathbf{B}} \mathbf{N}
$$

where $\widehat{\mathbf{B}}=\sum_{m=1}^{n}\left(\mathbf{B}_{0}^{m}\right)$ is a $2 \times 9$ constant matrix obtained from (14) and (20) and $\widehat{\mathbf{B}}$ can be written in the following form:

$$
\widehat{\mathbf{B}}=\left[\begin{array}{lllllllll}
\widehat{\alpha}_{11} & \widehat{\beta}_{11} & \widehat{\alpha}_{12} & \widehat{\alpha}_{13} & \widehat{\beta}_{12} & \widehat{\beta}_{13} & \widehat{\gamma}_{11} & \widehat{\gamma}_{12} & \widehat{\gamma}_{13} \\
\widehat{\alpha}_{21} & \widehat{\beta}_{21} & \widehat{\alpha}_{22} & \widehat{\alpha}_{23} & \widehat{\beta}_{22} & \widehat{\beta}_{23} & \widehat{\gamma}_{21} & \widehat{\gamma}_{22} & \widehat{\gamma}_{23}
\end{array}\right] .
$$

For convenience, the following definitions are introduced:

$$
\begin{aligned}
& \widehat{k}_{1 i}=m_{x} k_{1 i}, \\
& \widehat{k}_{2 i}=m_{y} k_{2 i},
\end{aligned}
$$

where $i=1,2,3 ; k=\alpha, \beta, \gamma$.

Equations (11) can be transformed into the following state vector equation by substituting $F_{d, x}$ and $F_{d, y}$ :

$$
\dot{\mathbf{x}}(t)-\mathbf{A x}(t)-\mathbf{C} \mathbf{x}(t-\tau)=\mathbf{F},
$$

where $\mathbf{x}$ is the state vector defined as $\mathbf{x}=\left[x_{1}, x_{2}, x_{3}, x_{4}\right]=$ $\left[\dot{x}_{p}, x_{p}, \dot{y}_{p}, y_{p}\right]^{\mathrm{T}}$.

Detailed expressions of the coefficient matrices of the state vectors ( $\mathbf{A}$ and $\mathbf{C}$ ) and the nonlinear forcing vector $(\mathbf{F})$ are given in Appendix B. 


\section{Linear Stability Analysis}

The linear stability of the ball-end milling system considered in this study can be determined by solving the eigenproblem composed of its linearized version. The characteristic equation derived from (25) takes the following form:

$$
\left|\lambda \mathbf{I}-\mathbf{A}-e^{-\lambda \tau} \mathbf{C}\right|=0,
$$

where $\lambda$ is the eigenvalue of the linearized system and symbol $|\cdot|$ indicates the determinant.

A necessary and sufficient condition for the system to be asymptotically stable is that the infinite number of characteristic exponents must have negative real part [26]. Because stability analysis requires only the sign of the real part of the rightmost eigenvalue, it is not necessary to compute the characteristic exponents. Accordingly, only the pure imaginary eigenvalues $(\lambda=i \omega,-i \omega)$ associated with a specific operating condition are needed to determine the stability boundaries dividing the stable and unstable regions.

Several analytical, semianalytical, and numerical methods have been used to obtain the stability boundary (lobe) diagram for ball-end milling systems. In this study, an analytical approach using the single-frequency solution [16-19, 27] was used. Note that the single-frequency solution was used because only machining with high radial immersion was considered in this study. This method [19] is briefly introduced in this section. First, the following determinant equation is considered:

$$
|\mathbf{I}+\mu \Phi|=0,
$$

where $\mu$ is the complex eigenvalue:

$$
\begin{aligned}
& \mu=-\delta_{z}\left(1-e^{-i \omega_{c} \tau}\right)=\mu_{R}+i \mu_{I}, \\
& \boldsymbol{\Phi}=\left[\begin{array}{ll}
\widehat{\alpha}_{11} & \widehat{\beta}_{11} \\
\widehat{\alpha}_{21} & \widehat{\beta}_{21}
\end{array}\right] \mathbf{G}\left(i \omega_{c}\right),
\end{aligned}
$$

where $\mathbf{G}$ is the transfer function matrix identified at the cutter-workpiece contact zone.

The critical element thickness can be calculated as follows [15-19]:

$$
\delta_{z c}=-\frac{1}{2} \mu_{R}\left(1+\left(\frac{\mu_{I}}{\mu_{R}}\right)^{2}\right) .
$$

The corresponding spindle speed $\Omega$ and tooth passing period can be determined as follows [16-19]:

$$
\tau=\frac{1}{\omega_{c}}\left(\pi-2 \tan ^{-1}\left(\frac{\mu_{I}}{\mu_{R}}\right)+2 k \pi\right), \quad \Omega=\frac{60}{N \tau} .
$$

Note that instead of attempting to obtain the critical depth of cut $a p_{c}=n \delta_{z c}$ directly, we first obtain the critical element thickness $\delta_{z c}$ in (30). The procedure for determining $a p_{c}$ is described in [18] and can be briefly summarized as follows. To solve (27), to determine the critical axial depth of cut, the number of the axial disks $n$ must be known a priori to construct the matrix $\Phi$. Therefore, a guessed value of the number of axial disks $n$ is used as a starting value to solve (27), from which a new value of the critical axial depth of cut is obtained. This procedure is repeated iteratively until it converges to a solution. Finally, the stability lobe diagram can be obtained by plotting the converged critical axial depth of cut with respect to the corresponding spindle speed.

\section{Normal Form Using the MMS near the Equilibrium State}

A bifurcation analysis was performed using MMS to investigate the local dynamic characteristics of the ball-end milling system near the stability boundaries, for which MMS is used as a perturbation technique. MHB is subsequently used to investigate the system's limit cycle behavior.

The time scales and the associated time derivatives are first defined as usual, such that

$$
\begin{aligned}
& T_{k}=\varepsilon^{k} t \quad \text { for } k=0,1,2, \ldots, \\
& \frac{\mathrm{d}}{\mathrm{d} t}=D_{0}+\varepsilon D_{1}+\varepsilon^{2} D_{2}+\cdots,
\end{aligned}
$$

where $\varepsilon$ is a small perturbation used as a bookmark in this study.

The present state can be expanded in the following form:

$$
\begin{aligned}
\mathbf{x}(t)= & \varepsilon \mathbf{x}^{(1)}\left(T_{0}, T_{2}\right)+\varepsilon^{2} \mathbf{x}^{(2)}\left(T_{0}, T_{2}\right) \\
& +\varepsilon^{3} \mathbf{x}^{(3)}\left(T_{0}, T_{2}\right) .
\end{aligned}
$$

Note that the terms of $T_{1}$ in (33) were intentionally omitted because there remained only trivial secular terms in the equation of $O\left(\varepsilon^{2}\right)$, which naturally resulted in $D_{1} A_{1}=$ $D_{1} A_{2}=0$. The delayed state can be expanded using a Taylor series, such that

$$
\begin{aligned}
\mathbf{x}(t-\tau)= & \varepsilon \mathbf{x}^{(1)}\left(T_{0}-\tau, T_{2}-\varepsilon^{2} \tau\right) \\
& +\varepsilon^{2} \mathbf{x}^{(2)}\left(T_{0}-\tau, T_{2}-\varepsilon^{2} \tau\right) \\
& +\varepsilon^{3} \mathbf{x}^{(3)}\left(T_{0}-\tau, T_{2}-\varepsilon^{2} \tau\right) \\
= & \varepsilon \mathbf{x}_{\tau}^{(1)}+\varepsilon^{2} \mathbf{x}_{\tau}^{(2)}+\varepsilon^{3}\left(\mathbf{x}_{\tau}^{(3)}-\tau D_{2} \mathbf{x}_{\tau}^{(1)}\right),
\end{aligned}
$$

where $\mathbf{x}_{\tau}^{(k)}=\mathbf{x}^{(k)}\left(T_{0}-\tau, T_{2}\right)(k=1,2,3)$.

The limiting depth of cut $a p$ can be considered the sum of the linear critical depth of cut $a p_{c}$ at the stability lobe and the perturbed depth of cut $a p_{\gamma}$; that is,

$$
a p=a p_{c}+\varepsilon^{2} a p_{\gamma} .
$$

When the number of axial directional discrete members (cut in the shape of thin disks of equal height) is determined, the element thickness $\delta_{z}$ can be obtained as the sum of its linear portion $\left(\delta_{z c}\right)$ and perturbed portion $\left(\delta_{z \gamma}\right)$; namely,

$$
\delta_{z}=\delta_{z c}+\varepsilon^{2} \delta_{z \gamma}=\frac{a p_{c}}{n}+\varepsilon^{2} \frac{a p_{\gamma}}{n} .
$$


By substituting (33)-(36) into (25) and then equating the coefficients of like powers of $\varepsilon$, the following differential equations are obtained up to the third order of $\varepsilon$ :

$$
\begin{aligned}
& \varepsilon^{1}: D_{0} \mathbf{x}^{(1)}-\mathbf{A}_{c} \mathbf{x}^{(1)}-\mathbf{C}_{c} \mathbf{x}_{\tau}^{(1)}=0, \\
& \varepsilon^{2}: D_{0} \mathbf{x}^{(2)}-\mathbf{A}_{c} \mathbf{x}^{(2)}-\mathbf{C}_{c} \mathbf{x}_{\tau}^{(2)}=\mathbf{F}^{(2)}, \\
& \varepsilon^{3}: D_{0} \mathbf{x}^{(3)}-\mathbf{A}_{c} \mathbf{x}^{(3)}-\mathbf{C}_{c} \mathbf{x}_{\tau}^{(3)} \\
& \quad=\mathbf{F}^{(3)}-D_{2} \mathbf{x}^{(1)}+\mathbf{A}_{\gamma} \mathbf{x}^{(1)}+\mathbf{C}_{\gamma} \mathbf{x}_{\tau}^{(1)}-\mathbf{C}_{c} \tau D_{2} \mathbf{x}_{\tau}^{(1)},
\end{aligned}
$$

where $\mathbf{A}_{c}$ and $\mathbf{C}_{c}$ denote the coefficient matrices of the current and delayed state vectors with the linear critical depth of cut $a p_{c}, \mathbf{A}_{\gamma}$ and $\mathbf{C}_{\gamma}$ denote the coefficient matrices of the perturbed depth of cut $a p_{\gamma}$, and $\mathbf{F}^{(2)}$ and $\mathbf{F}^{(3)}$ denote the terms of the second and third orders of $\varepsilon$, respectively, in the nonlinear forcing vector $\mathbf{F}$ (see Appendix B for their detailed expressions).

According to [26], the harmonic solution of (37) can be assumed to be as follows:

$$
\mathbf{x}^{(1)}=\mathbf{X}_{1} A_{1}\left(T_{2}\right) e^{i \omega_{c} T_{0}}+\mathbf{X}_{2} A_{2}\left(T_{2}\right) e^{-i \omega_{c} T_{0}},
$$

where $\omega_{c}$ is the chatter (angular) frequency, $\mathbf{X}_{1}$ and $\mathbf{X}_{2}$ are the eigenvectors associated with the eigenvalues $\lambda_{1}=i \omega_{c}$ and $\lambda_{2}=-i \omega_{c}$, respectively, and

$$
\mathbf{X}_{k}=\left[\lambda_{k}, 1, \lambda_{k} \eta_{k}, \eta_{k}\right]^{\mathrm{T}}, \quad(k=1,2),
$$

where $\eta_{k}=\left(\lambda_{k}^{2}+2 \lambda_{k} \zeta_{x}+\omega_{x}^{2}-\delta_{z c} \alpha_{11}\left(1-e^{-\lambda_{k} \tau}\right)\right) / \delta_{z c} \beta_{11}(1-$ $\left.e^{-\lambda_{k} \tau}\right)$.

The substitution of (40) into (38) yields the following:

$$
\begin{aligned}
D_{0} \mathbf{x}^{(2)}-\mathbf{A}_{c} \mathbf{x}^{(2)}-\mathbf{C}_{c} \mathbf{x}_{\tau}^{(2)} \\
=\mathbf{Q}_{0}^{(2)}\left(A_{1}, A_{2}\right)+\mathbf{Q}_{1}^{(2)}\left(A_{1}, A_{2}\right) e^{i \omega_{c} T_{0}} \\
\quad+\mathbf{Q}_{3}^{(2)}\left(A_{1}, A_{2}\right) e^{i 2 \omega_{c} T_{0}}+\mathrm{cc} \\
=\mathbf{Q}_{0}^{(F 2)} A_{1} A_{2}-\mathbf{Q}_{2}^{(F 2)} A_{1}^{2} e^{i 2 \omega_{c} T_{0}}+\mathrm{cc},
\end{aligned}
$$

where $\mathrm{cc}$ denotes the complex conjugate of the preceding term. form:

The solution of (42) can now be expressed in the following

$$
\mathbf{x}^{(2)}=\mathbf{P}_{2}^{(2)} A_{1}^{2} e^{2 i \omega_{c} T_{0}}+\mathbf{P}_{0}^{(2)} A_{1} A_{2}+\mathbf{P}_{-2}^{(2)} A_{2}^{2} e^{-2 i \omega_{c} T_{0}}
$$

where $\mathbf{P}_{2}^{(2)}, \mathbf{P}_{0}^{(2)}$, and $\mathbf{P}_{-2}^{(2)}$ are the shape vectors of complex harmonics (see Appendix B for details). The substitution of (40) and (43) into (39) yields the following:

$$
\begin{aligned}
& D_{0} \mathbf{x}^{(3)}-\mathbf{A}_{c} \mathbf{x}^{(3)}-\mathbf{C}_{c} \mathbf{x}_{\tau}^{(3)} \\
& \quad=\mathbf{Q}_{1}^{(3)}\left(A_{1}, A_{2}\right) e^{i \omega_{c} T_{0}}+\mathbf{Q}_{3}^{(3)}\left(A_{1}, A_{2}\right) e^{i 3 \omega_{c} T_{0}}+\mathrm{cc} .
\end{aligned}
$$

Taking the forcing vector associated with the secular components, including $e^{i \omega_{c} T_{0}},(44)$ becomes as follows:

$$
\begin{aligned}
& D_{0} \mathbf{x}^{(3)}-\mathbf{A}_{c} \mathbf{x}^{(3)}-\mathbf{C}_{c} \mathbf{x}_{\tau}^{(3)} \\
& =\left[-\left(\mathbf{I}+\tau \mathbf{C}_{c} e^{-i \omega_{c} \tau}\right) \mathbf{X}_{1} D_{2} A_{1}\right. \\
& \left.+\left(\mathbf{A}_{\gamma}+\mathbf{C}_{\gamma} e^{-i \omega_{c} \tau}\right) \mathbf{X}_{1} A_{1}+\mathbf{Q}_{1}^{(F 3)} A_{1}^{2} A_{2}\right] e^{i \omega_{c} T_{0}} \\
& =\left[\mathbf{Q}_{1}^{(D)} D_{2} A_{1}+\mathbf{Q}_{1}^{(\gamma)} A_{1}+\mathbf{Q}_{1}^{(C)} A_{1}^{2} A_{2}\right] e^{i \omega_{c} T_{0}},
\end{aligned}
$$

where $\mathbf{Q}_{1}^{(F 3)}$ is the matrix of coefficient vectors for the complex harmonic terms originating from $\mathbf{F}^{(3)}$ (see Appendix B for details).

The solvability condition of the third order of $\varepsilon$ yields the following modulation equation in the complex form:

$$
\dot{A}_{1}=\left(\vartheta_{r 1}+i \vartheta_{i 1}\right) \delta_{z \gamma} A_{1}+\left(\vartheta_{r 2}+i \vartheta_{i 2}\right) A_{1}^{2} A_{2},
$$

where $\vartheta_{r 1}, \vartheta_{i 1}, \vartheta_{r 2}$, and $\vartheta_{i 2}$ are the real constants (as given in Appendix B).

Using the polar-form expressions of the amplitudes, such as

$$
\begin{aligned}
& A_{1}=\frac{1}{2} a e^{i \psi}, \\
& A_{2}=\frac{1}{2} a e^{-i \psi},
\end{aligned}
$$

the following two modulation equations can be obtained:

$$
\begin{array}{r}
\dot{a}=\vartheta_{r 1} \delta_{z \gamma} a+\frac{1}{4} \vartheta_{r 2} a^{3}, \\
a \dot{\psi}=\vartheta_{i 2} \delta_{z \gamma} a+\frac{1}{4} \vartheta_{i 2} a^{3} .
\end{array}
$$

The stationary limit cycles bifurcating from the Hopf points can be obtained by applying the steady-state condition to (48). The resulting limit cycle becomes subcritical when the signs of both $\vartheta_{r 1}$ and $\vartheta_{r 2}$ are the same; otherwise, it becomes supercritical [28].

\section{Periodic Solutions of the Nonlinear Ball- End Milling System Using MHB}

As briefly noted in the previous section, MMS is a semianalytical perturbation method used to examine the local nonlinear dynamic characteristics of DDEs [9]. Although it is a useful and effective method that can provide physical insights into the complicated dynamic nature of the present nonlinear ball-end milling system (as explained in Section 4), MMS is intrinsically limited in that the nonlinearity must be small enough to obtain rapid convergence of the associated asymptotic solutions [28]. MHB [21] is known to be very useful in investigating steady-state periodic behaviors of nonlinear systems if they appear. 
To construct the perturbation solutions of (11), we note that they can be expressed as $n$th order Fourier series:

$$
\begin{aligned}
& x_{p}=\sum_{k=0}^{N} p_{x k} \cos (k \widehat{\omega} t)+q_{x k} \sin (k \widehat{\omega} t), \\
& y_{p}=\sum_{k=0}^{N} p_{y k} \cos (k \widehat{\omega} t)+q_{y k} \sin (k \widehat{\omega} t),
\end{aligned}
$$

where $q_{x 0}=q_{y 0}=0$ and $q_{x 1}=0$.

Substituting (49) into the governing differential equations (11) and equating the coefficient of each harmonic term on both sides of the resulting equations yield $2 \times(2 N+1)$ nonlinear algebraic equations with unknown variables for the fundamental oscillation frequency $(\widehat{\omega})$ and the harmonic coefficients $\left(p_{x k}, q_{x k}, p_{y k}\right.$, and $\left.q_{y k}\right)$. The resulting algebraic equations were solved using the symbolic manipulator MAPLE [29]. Because of the lengthy expressions in these equations, we only show the numerical results using the figures in the next section.

\section{Results and Discussion}

6.1. Tool and Dynamic Parameters. The ball-end milling system introduced in [19] was employed for illustration purposes in the present study. The values of the associated tool and dynamic parameters are given in [18] as follows:

tool parameters: $N_{f}=2$ teeth, $R_{0}=6 \mathrm{~mm}, i_{0}=30^{\circ}$, and rake angle $=8^{\circ}$;

workpiece: AISI 1050 steel;

Modal 1: $f_{n 1}=2983.8 \mathrm{~Hz}, \zeta_{1}=0.01550$, and $m_{1}=$ $0.0769 \mathrm{~kg}$;

Modal 2: $f_{n 2}=4022.2 \mathrm{~Hz}, \zeta_{2}=0.01846$, and $m_{2}=$ $0.0629 \mathrm{~kg}$.

Note that the modal parameters listed above are associated with the two fundamental normal modes in the system's principal directions $(x$ and $y$ ).

\subsection{Cutting Force Coefficients and Prediction of the Cutting} Force. The cutting force coefficients of the linear model were calculated using the well-known mechanics of orthogonal milling process data $[5,19]$ as follows:

$$
\begin{aligned}
& \text { chip thickness ratio: } r=0.40+0.0005 \mathrm{~V}+0.6 \mathrm{~s}, \\
& \text { friction angle: } \beta_{f}=26.8-0.0313 \mathrm{~V}+11.77 \mathrm{~s}(\mathrm{deg}), \\
& \text { shear stress: } T_{\text {shear }}=450.3+0.4 \mathrm{~V}+227.5 \mathrm{~s}(\mathrm{MPa}),
\end{aligned}
$$

where $s(\mathrm{~mm})$ and $V(\mathrm{~mm} / \mathrm{min})$ are the static uncut chip thickness and cutting speed, respectively.

The cutting force components in the $-x,-y$, and $-z$ axes can be calculated using the method introduced in [5].

When a nonlinear ball-end milling model is considered, the procedure introduced in [1] can be employed to calculate the nonlinear cutting forces. The empirical cutting force coefficients in (3) are better calibrated using an efficient method,
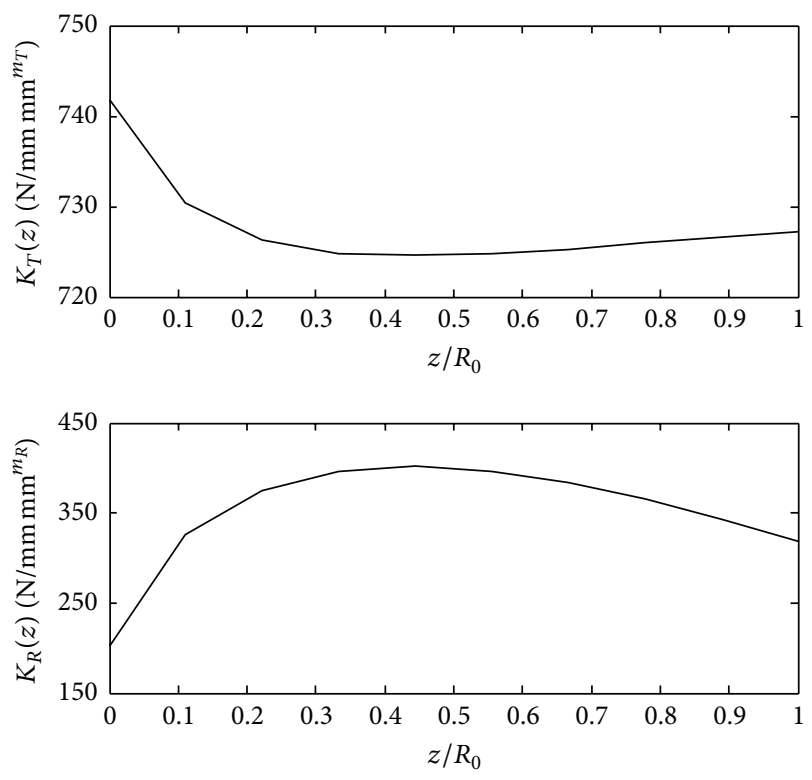

Figure 2: Plots of $K_{T}(z)$ and $K_{R}(z)$ along the cutter axis.

namely, processing of the instantaneous cutting forces [2]. For the ball-end milling system under consideration, the following exponents of the chip thickness were employed: $m_{T}=0.7239, m_{R}=0.8203$. The corresponding discrete values of the cutting-mechanics parameters $K_{T}(z)$ and $K_{R}(z)$ along the cutter axis are plotted in Figure 2.

Although the nonlinear force model cannot be used to predict the cutting force along the $z$-axis (the tool axis), because a ball-end mill tool is usually assumed to be rigid in the direction of the tool axis, the cutting force in the $z$ direction can be ignored and is not considered here. Figure 3 shows a comparison between the two static cutting force models (linear and nonlinear models) for a slotting cut with the feed per tooth $f_{z}=0.05 \mathrm{~mm} /$ tooth. The relationship between the cutting force and the chip thickness employed in the linear force model of the present study can briefly be summarized by the steps explained in Appendix C. It should be noted that the linear and nonlinear force models used in this study are different in origin. The linear force model, represented by (C.6) in Appendix C, is not the model that can be obtained from the linearization of the nonlinear force model (1)-(5), which results in the difference in the stability lobes shown in Figure 4 . This figure shows that the difference between these two models is not significant. Therefore, the use of either the linear or the nonlinear force model to predict the static cutting force (without vibration) in the ball-end milling process is acceptable. Note that the static cutting forces in Figure 3 are obtained at steady-state conditions.

6.3. Stability Lobes and the Associated Hopf Bifurcations. The stability lobes can be obtained in terms of the spindle speed $(\Omega)$ and the depth of cut $(a p)$. Figure 4 shows stability lobes obtained with the linear force model, which are also shown in [13], and those obtained with the linearized nonlinear force model with the following values for the feed per tooth: 


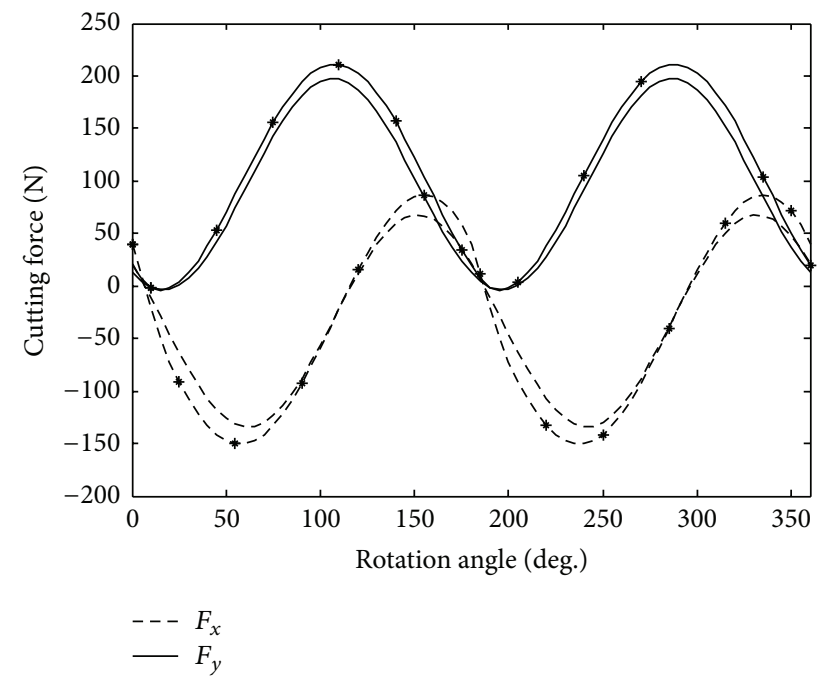

FIGURE 3: Comparison of the nonlinear cutting force model (stars) and the linear cutting force model (no markers).

$f_{z}=0.05 \mathrm{~mm} /$ tooth (Figure $4(\mathrm{a})$ ) and $f_{z}=0.1 \mathrm{~mm} /$ tooth (Figure 4(b)). In Figure 4, the upper and lower domains of the stability lobes correspond to the unstable and stable regions, respectively. The stability of the system was also validated by comparison with experimental data presented in [13]. When the depth of cut $(a p)$ is less than the critical depth of cut $\left(a p_{c}\right)$, the system is stable at equilibrium. However, when $a p>a p_{c}$, the system becomes unstable. Therefore, in the vicinity of equilibrium, the ball-end milling system will exhibit convergent (stable) and divergent (unstable) behaviors before and after the linear critical depth of cut, respectively. As is evident in Figure 4, the stability lobes obtained with the linear force model would be similar for other values of the feed per tooth. In contrast, the stability lobes predicted using the linearized nonlinear force model are highly dependent on the value of the feed per tooth. This difference arises from the fact that the stability lobes obtained with the linearized nonlinear force model are greatly affected by the noninteger power functions and changes in the cutting force coefficients (here, the effect of the noninteger power functions is the strongest). On the other hand, when the linear force model is used, the stability lobe is only affected by the changes in the cutting force coefficients, and even this change is very small for different values of the feed per tooth.

In this case study, two dominant modes of the system were considered. The stability lobes depicted in Figure 5, which were obtained using the linearized nonlinear force model, are the same as those shown in Figure 4(a), with the exception of the lines, which represent the contribution of each mode to the stability lobes. Figure 5 is obtained by separately considering the contribution of each mode for the stability lobes through the transfer function matrix $\mathbf{G}$ in (29). To create this figure, initially, only the first mode is considered to calculate the transfer function matrix G. With the transfer function matrix $\mathbf{G}$ obtained, the stability lobes for the first mode are obtained (solid line). A similar process is used to obtain the stability lobes for the second mode (dashed line). In Figure 5,
Mode $k(k=1,2)$ means that the result was obtained using only the $k$ th dominant mode in the stability computation. The region with a gray background represents the stable region obtained from consideration of the contributions of both modes.

A1 and A2 points are Hopf bifurcation points separately characterized by the 1st and 2 nd modes, respectively, whereas A3 point is a double-Hopf bifurcation point characterized by the combination of both modes. The local dynamic characteristics of the nonlinear ball-end milling system in the vicinity of the stability boundaries were investigated through a bifurcation analysis using MMS. Figure 6 shows the bifurcation diagrams for the amplitudes of the limit cycles computed along the $x$-axis $\left(a_{x 2}\right)$ with respect to the axial depth of cut $(a p)$, evaluated at the spindle speeds corresponding to A1 (Figure 6(a)) and A2 (Figure 6(b)) marked the same as in Figure 5. Note that the amplitudes of the limit cycles in Figure 6(a) were obtained only with the first mode because the effect of the second mode on the amplitude is negligibly small. On the other hand, the amplitudes of the limit cycles in Figure 6(b) were obtained only with the second mode because, in this case, the effect of the first mode on the amplitude is negligibly small. Note that the resulting amplitudes of the limit cycles in Figure 6 are small enough to justify the use of the MMS.

As shown in Figure 6, when the axial depth of cut (ap) changes from the stable region to the unstable region along the arrowed path in the figure, the ball-end milling system tends to lose stability at the critical equilibrium point. The amplitudes of the limit cycles bifurcate similarly at both critical equilibrium points (indicated as A1 and A2 in Figure 5), resulting in the subcritical Hopf bifurcations. As the axial depth of cut $(a p)$ increases, the stable equilibrium is transformed into an unstable equilibrium. The attraction region near the stable equilibrium point is bounded by an unstable limit cycle branch. The chatter amplitude of the ball-end milling system diverges at the critical points (A1 and A2 in Figure 6) and moves away from the vicinity of the equilibrium point. It can be deduced that the actual (nonlinear) ballend milling system may not be readily controllable at these critical points over the parametric domain considered in this study. In contrast to the use of the linear cutting force model, when the nonlinear cutting force model is considered, it is shown that a large-amplitude chatter vibration can occur even when the depth of cut is less than the critical depth of cut. This means that, in the region underneath the stability lobe, when the chip thickness approaches its critical value along the linear stability boundary, unstable oscillations will appear around the locally stable stationary cutting state. For such cases, other attractors related to another type of nonlinearity exist [30], which involve the loss of contact between the tool and workpiece. In practical systems, right after the vibration amplitude reaches the critical value, the tool tends to leave the workpiece material over part of the chatter cycle (i.e., loss of contact occurs). From the physical perspective, the material chipping process is discontinuous. From the geometrical perspective, however, the pattern of the variation in the thickness of the removed chips will be very complicated. A stationary cutting process coexists in the bistable region (or 


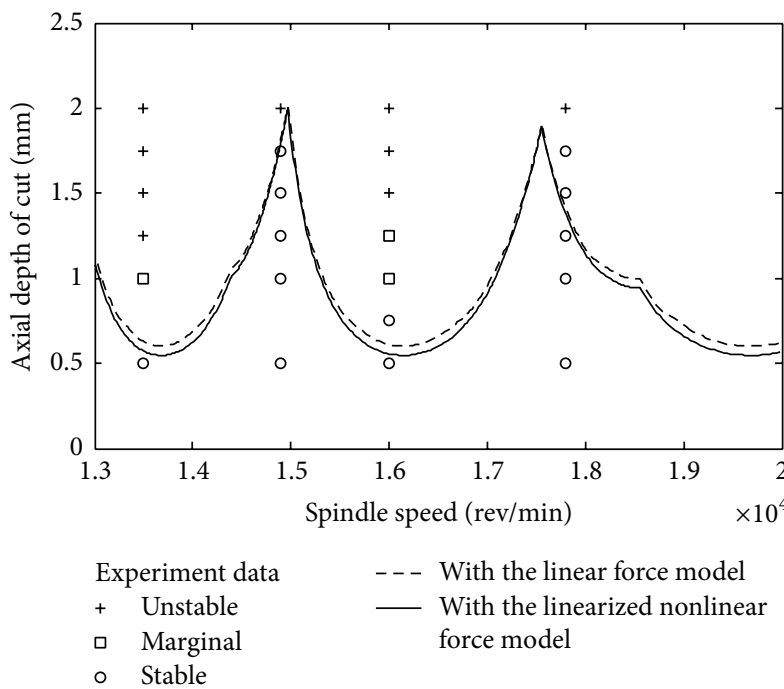

(a)

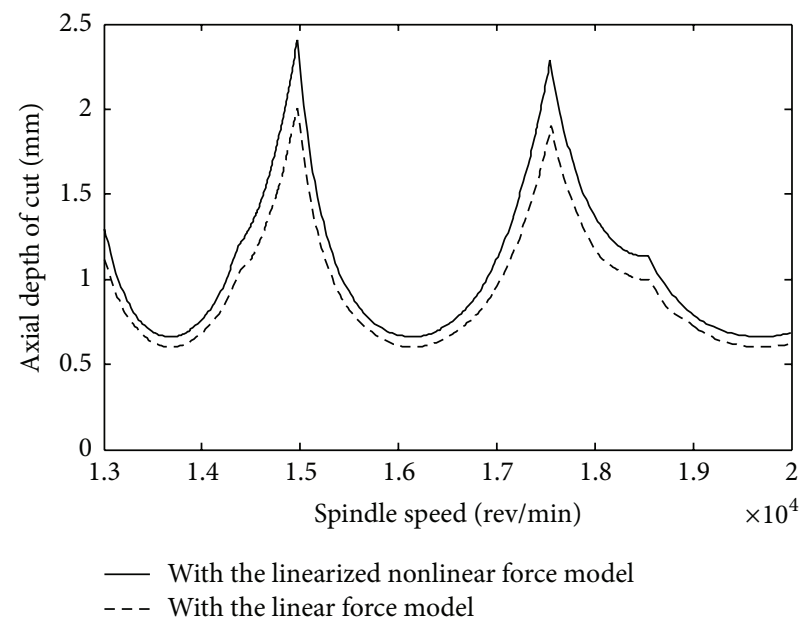

(b)

FIgURE 4: Stability lobes of ball-end milling system for (a) $f_{z}=0.05 \mathrm{~mm} /$ tooth and (b) $f_{z}=0.1 \mathrm{~mm} /$ tooth and experimental data [19].

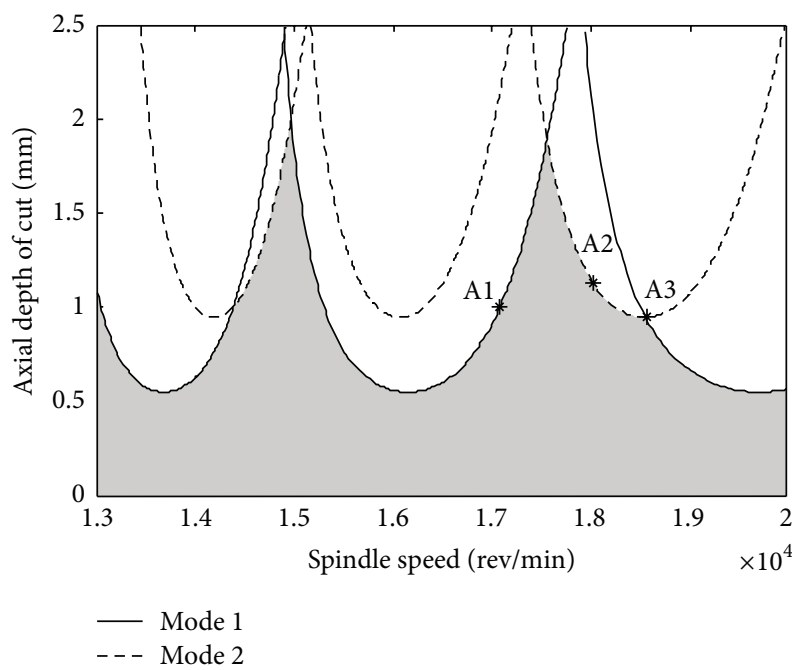

FIGURE 5: Stability lobes of ball-end milling system obtained with consideration of the contributions of two modes.

conditionally stable region) with a large-amplitude nonlinear oscillation, in general, accompanied by the loss of contact [31]. It should be noted that the loss of contact (and the associated bistable region) needs to be incorporated in the model for better practical modeling of the ball-end milling process.

Note that, unlike points $\mathrm{A} 1$ and $\mathrm{A} 2$, a double-Hopf bifurcation occurs at a specific point (A3), a crossover point of the two neighbouring lobes. In general, to obtain bifurcation diagrams for the actual amplitudes of the limit cycles corresponding to such a specific point (A3), the dimension of the system needs to be doubled and thus extended to the four dimensions in the present model. This is out of the scope of this study and thus not performed. However, this point can be readily obtained by finding the intersection point of the two neighbouring stability lobes separately characterized by two different modes.

Figure 7 shows a comparison of the bifurcation diagrams obtained with MMS and MHB with three harmonic terms. In Figure 7, the region between the two dotted lines corresponds to the parametric domain shown in Figure 6, with the difference that the depth of cut $a p$ is replaced by a perturbed depth of cut $a p_{\gamma}$. As clearly shown in this figure, the results obtained using MMS and MHB are in good agreement, particularly when the amplitude of the limit cycle is small.

Direct numerical time integrations were also performed for (25) to validate the limit cycle solutions obtained using the MHB. The numerical time integration scheme used for (25) is a direct integration approach using the subroutine dde23 [32] provided by MATLAB [33]. For numerical integrations, the time history during $t \in[-\tau, 0]$ is given $a$ priori with the hypothetical steady-state solution of a period obtained from the MHB applied to the unstable limit cycle that would finally arrive at a converged trivial solution as evidenced by Figure 8 . Note that the MHB can yield a hypothetical periodic solution at the estimation point (point $P$ in Figure 7 ).

\section{Conclusions}

A dynamic model for a ball-end milling process that considers cutting force nonlinearities and the regenerative chatter effect is presented in this paper. The stability lobe diagram obtained using the linearized nonlinear cutting force model was compared to the one obtained using the linear cutting force model. A series of nonlinear analyses was performed to investigate the nonlinear dynamic behavior of the ball-end milling system in the case of high immersion of the cutting tool in the workpiece.

For convenience, the cutting force was expanded to a Taylor series up to the third order. Using only the zeroth order of the Fourier series, the system can be approximated by a 


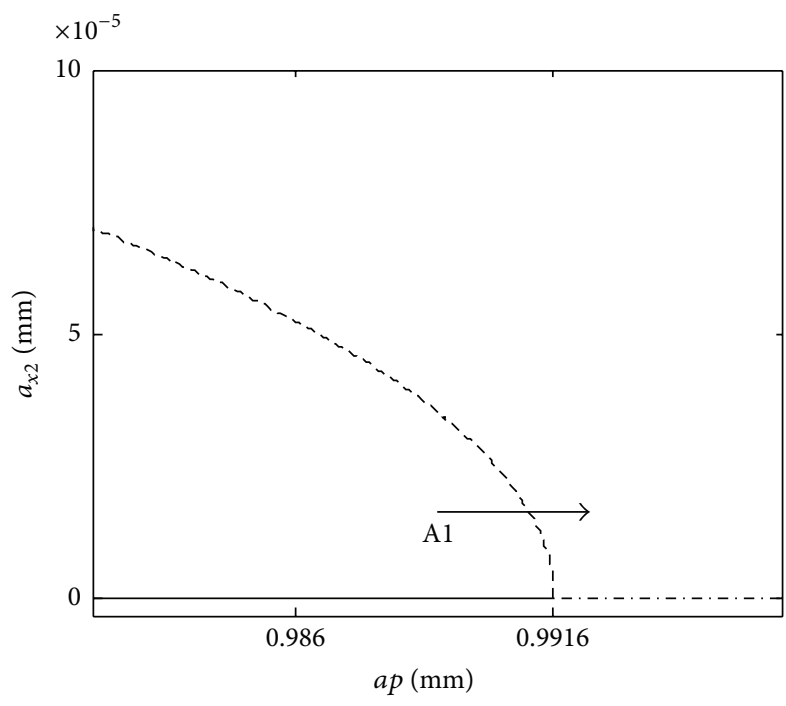

(a)

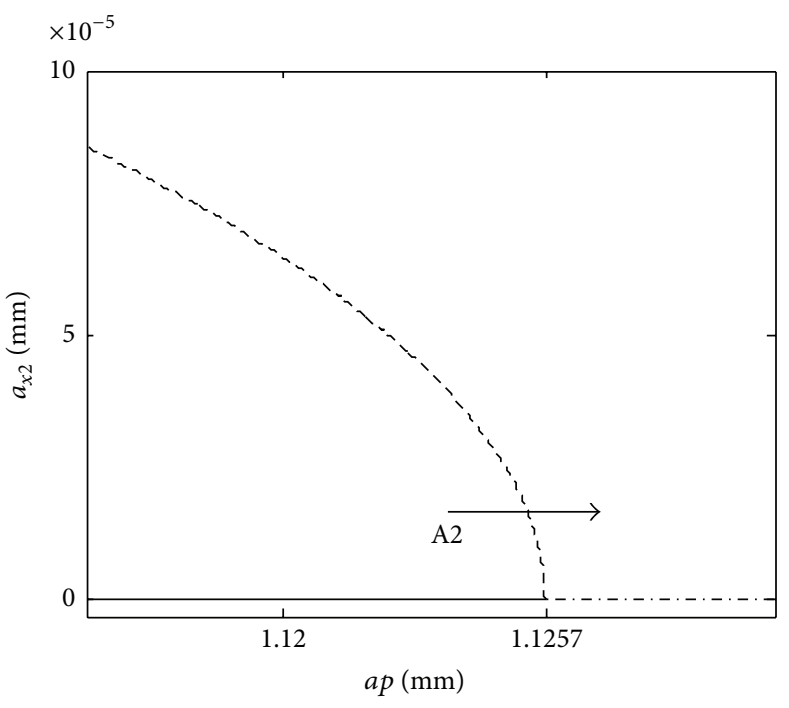

(b)

FIGURE 6: Limit cycle branches bifurcating from Hopf bifurcation points (A1 and A2 in Figure 5).

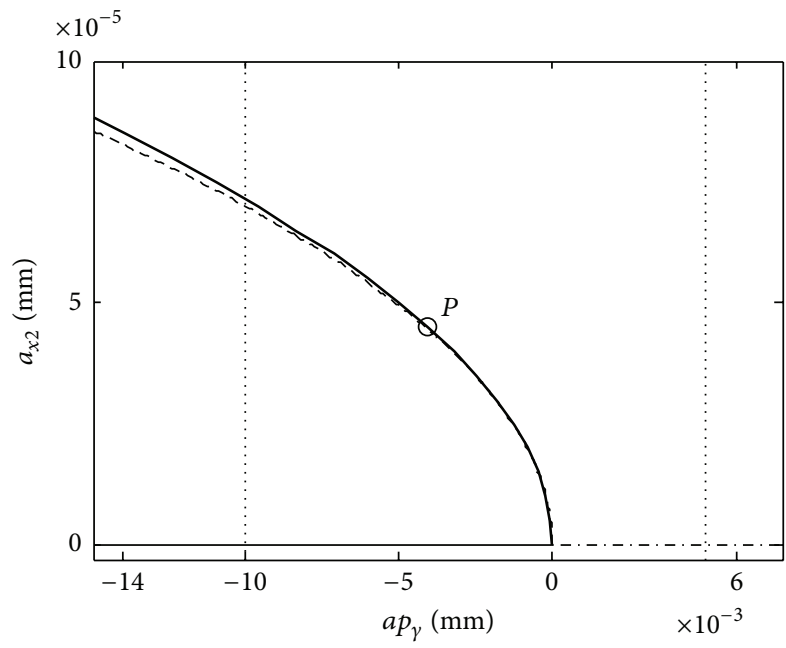

FIGURE 7: Comparison of the bifurcation diagrams obtained using the MMS (dashed line) and MHB (thick solid line).

set of nonlinear autonomous DDEs. Based on this model, stability and bifurcation analyses were performed to examine the predicted nonlinear local chatter behavior. The following conclusions can be drawn from the results of these analyses.

(1) The stability lobe diagram obtained using the linearized nonlinear cutting force model is highly dependent on the feed per tooth, while that obtained using the linear model does not depend significantly on the feed per tooth.

(2) A bifurcation analysis was performed near the critical equilibrium point of the ball-end milling system

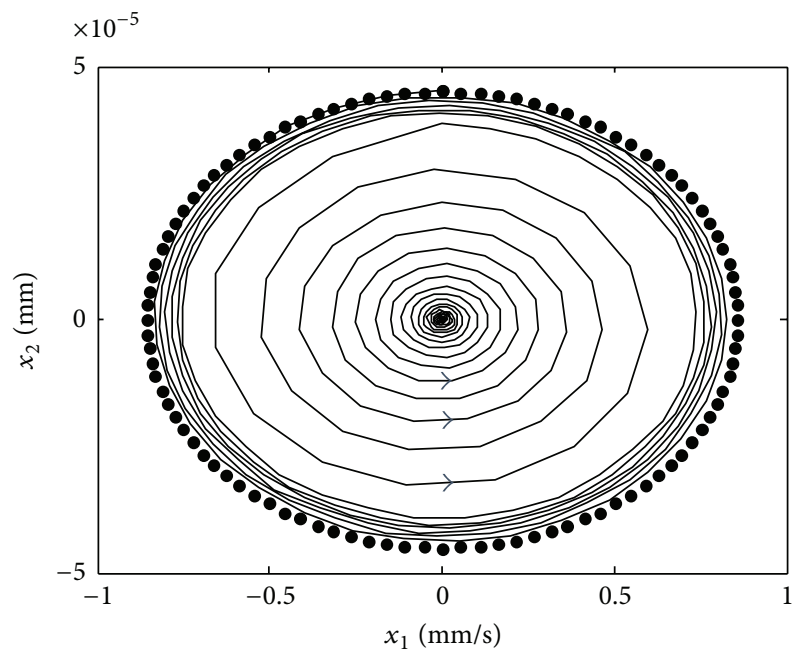

FIGURE 8: Phase-plane representations of an unstable limit cycle solution: the solid line represents the time histories obtained using the direct integrations (with varying time step), and the dashed line represents the hypothetical periodic time histories obtained using the MHB estimated at the unstable limit cycle point. The arrow shows a brief behavioural time trace of the unstable limit cycle solution.

using MMS and further investigated using MHB to analyze its local chatter behavior. The criticality of the Hopf bifurcations that occur can be analytically determined using these approaches. Subcritical Hopf bifurcations were observed to originate from the two points investigated in this study (A1 and A2). As a result, it was observed that the unstable limit cycle solution generated by the subcritical Hopf bifurcation at the equilibrium point tends to grow. It was also 
shown that, in contrast to the use of the linear cutting force model, when the nonlinear cutting force model is used, large-amplitude chatter vibration can occur as result of an external disturbance, even when the depth of cut is less than the critical depth of cut.

(3) Because the simple approximation of the cutting forces obtained using the zeroth order of the Fourier series cannot be used to examine the global chatter behaviors of this ball-end milling system, a higherorder expansion needs to be employed for this purpose. This will be performed in a future study.

\section{Appendices}

\section{A. Detailed Expressions of the Symbols Used in the Governing Differential Equations}

A.1. Parameter Matrices of $\zeta, \boldsymbol{\omega}, \mathbf{M}$, and $\mathbf{F}_{s}$ in (10). Consider

$$
\begin{aligned}
\boldsymbol{\zeta} & =\left[\begin{array}{cc}
2 \zeta_{x} & 0 \\
0 & 2 \zeta_{y}
\end{array}\right], \\
\boldsymbol{\omega} & =\left[\begin{array}{cc}
\omega_{x}^{2} & 0 \\
0 & \omega_{y}^{2}
\end{array}\right], \\
\mathbf{M} & =\left[\begin{array}{cc}
m_{x} & 0 \\
0 & m_{y}
\end{array}\right], \\
\mathbf{F}_{s} & =\delta_{z} \sum_{m=1}^{n} \sum_{i=1}^{N_{f}}\left[\begin{array}{ll}
-\cos \varphi_{i, m} & -\sin \varphi_{i, m} \\
\sin \varphi_{i, m} & -\cos \varphi_{i, m}
\end{array}\right]\left[\begin{array}{l}
K_{T, m} s_{i, m}^{m_{T}} \\
K_{R, m} s_{i, m}^{m_{R}}
\end{array}\right] .
\end{aligned}
$$

A.2. Taylor Series Expressions of Nonlinear Cutting Forces and Detailed Expressions of $a_{k}$ and $b_{k}(k=1, \ldots, 3)$ in (12). Consider

$$
\begin{aligned}
\mathrm{d} F_{T, i}(z, \varphi) & =K_{T}(z)\left[h_{i}(\varphi)\right]^{m_{T}} \mathrm{~d} z \\
& \cong\left(a_{0}+a_{1} u_{i, m}+a_{2} u_{i, m}^{2}+a_{3} u_{i, m}^{3}\right) \mathrm{d} z, \\
\mathrm{~d} F_{R, i}(z, \varphi) & =K_{R}(z)\left[h_{i}(\varphi)\right]^{m_{R}} \mathrm{~d} z \\
& \cong\left(b_{0}+b_{1} u_{i, m}+b_{2} u_{i, m}^{2}+b_{3} u_{i, m}^{3}\right) \mathrm{d} z, \\
a_{0} & =K_{T, m} s_{i, m}^{m_{T}}, \\
a_{1} & =K_{T, m} m_{T} s_{i, m}^{m_{T}-1}, \\
a_{2} & =\frac{1}{2} K_{T, m} m_{T}\left(m_{T}-1\right) s_{i, m}^{m_{T}-2},
\end{aligned}
$$

$$
\begin{aligned}
& a_{3}=\frac{1}{6} K_{T, m} m_{T}\left(m_{T}-1\right)\left(m_{T}-2\right) s_{i, m}^{m_{T}-3}, \\
& b_{0}=K_{R, m} s_{i, m}^{m_{R}}, \\
& b_{1}=K_{R, m} m_{R} s_{i, m}^{m_{R}-1}, \\
& b_{2}=\frac{1}{2} K_{R, m} m_{R}\left(m_{R}-1\right) s_{i, m}^{m_{R}-2}, \\
& b_{3}=\frac{1}{6} K_{R, m} m_{R}\left(m_{R}-1\right)\left(m_{R}-2\right) s_{i, m}^{m_{R}-3} .
\end{aligned}
$$

A.3. Detailed Expression of $\mathbf{B}^{i, m}$ in (14). Consider

$$
\begin{aligned}
& \mathbf{B}^{i, m} \\
& =\left[\begin{array}{lllllllll}
B_{11} & B_{12} & B_{13} & B_{14} & B_{15} & B_{16} & B_{17} & B_{18} & B_{19} \\
B_{21} & B_{22} & B_{23} & B_{24} & B_{25} & B_{26} & B_{27} & B_{28} & B_{29}
\end{array}\right], \\
& B_{11}=-\left(a_{1} \cos \varphi_{i}+b_{1} \sin \varphi_{i}\right) \sin \varphi_{i}, \\
& B_{12}=-\left(a_{1} \cos \varphi_{i}+b_{1} \sin \varphi_{i}\right) \cos \varphi_{i}, \\
& B_{13}=-\left(a_{2} \cos \varphi_{i}+b_{2} \sin \varphi_{i}\right) \sin ^{2} \varphi_{i}, \\
& B_{14}=-\left(a_{3} \cos \varphi_{i}+b_{3} \sin \varphi_{i}\right) \sin ^{3} \varphi_{i} . \\
& B_{15}=-\left(a_{2} \cos \varphi_{i}+b_{2} \sin \varphi_{i}\right) \cos ^{2} \varphi_{i}, \\
& B_{16}=-\left(a_{3} \cos \varphi_{i}+b_{3} \sin \varphi_{i}\right) \cos ^{3} \varphi_{i}, \\
& B_{17}=-2\left(a_{2} \cos \varphi_{i}+b_{2} \sin \varphi_{i}\right) \sin \varphi_{i} \cos \varphi_{i}, \\
& B_{18}=-3\left(a_{3} \cos \varphi_{i}+b_{3} \sin \varphi_{i}\right) \sin ^{2} \varphi_{i} \cos \varphi_{i}, \\
& B_{19}=-3\left(a_{3} \cos \varphi_{i}+b_{3} \sin \varphi_{i}\right) \sin \varphi_{i} \cos ^{2} \varphi_{i}, \\
& B_{21}=\left(a_{1} \sin \varphi_{i}-b_{1} \cos \varphi_{i}\right) \sin \varphi_{i}, \\
& B_{22}=\left(a_{1} \sin \varphi_{i}-b_{1} \cos \varphi_{i}\right) \cos \varphi_{i}, \\
& B_{23}=\left(a_{2} \sin \varphi_{i}-b_{2} \cos \varphi_{i}\right) \sin ^{2} \varphi_{i}, \\
& B_{24}=\left(a_{3} \sin \varphi_{i}-b_{3} \cos \varphi_{i}\right) \sin ^{3} \varphi_{i} \text {, } \\
& B_{25}=\left(a_{2} \sin \varphi_{i}-b_{2} \cos \varphi_{i}\right) \cos ^{2} \varphi_{i}, \\
& B_{26}=\left(a_{3} \sin \varphi_{i}-b_{3} \cos \varphi_{i}\right) \cos ^{3} \varphi_{i},
\end{aligned}
$$




$$
\begin{aligned}
& B_{27}=2\left(a_{2} \sin \varphi_{i}-b_{2} \cos \varphi_{i}\right) \sin \varphi_{i} \cos \varphi_{i} \\
& B_{28}=3\left(a_{3} \sin \varphi_{i}-b_{3} \cos \varphi_{i}\right) \sin ^{2} \varphi_{i} \cos \varphi_{i} \\
& B_{29}=3\left(a_{3} \sin \varphi_{i}-b_{3} \cos \varphi_{i}\right) \sin \varphi_{i} \cos ^{2} \varphi_{i} .
\end{aligned}
$$

\section{B. Detailed Expressions of the Symbols Used in MMS}

B.1. Coefficient Matrices of $\mathbf{A}$ and $\mathbf{C}$ and the Forcing Vector $\mathbf{F}$ in (25). Consider

$$
\begin{aligned}
& \mathbf{A}=\left[\begin{array}{cccc}
-2 \zeta_{x} & -\omega_{x}^{2}+\delta_{z} \alpha_{11} & 0 & \delta_{z} \beta_{11} \\
1 & 0 & 0 & 0 \\
0 & \delta_{z} \alpha_{21} & -2 \zeta_{y} & -\omega_{y}^{2}+\delta_{z} \beta_{21} \\
0 & 0 & 1 & 0
\end{array}\right] \\
& \mathbf{C}=\left[\begin{array}{cccc}
0 & -\delta_{z} \alpha_{11} & 0 & -\delta_{z} \beta_{11} \\
0 & 0 & 0 & 0 \\
0 & -\delta_{z} \alpha_{21} & 0 & -\delta_{z} \beta_{21} \\
0 & 0 & 0 & 0
\end{array}\right] \\
& \mathbf{F}=\left[\begin{array}{c}
\delta_{z}\left(\alpha_{12} \Delta_{x}^{2}+\alpha_{13} \Delta_{x}^{3}+\beta_{12} \Delta_{y}^{2}+\beta_{13} \Delta_{y}^{3}+\gamma_{11} \Delta_{x} \Delta_{y}+\gamma_{12} \Delta_{x}^{2} \Delta_{y}+\gamma_{13} \Delta_{x} \Delta_{y}^{2}\right) \\
0 \\
\delta_{z}\left(\alpha_{22} \Delta_{x}^{2}+\alpha_{23} \Delta_{x}^{3}+\beta_{22} \Delta_{y}^{2}+\beta_{23} \Delta_{y}^{3}+\gamma_{21} \Delta_{x} \Delta_{y}+\gamma_{22} \Delta_{x}^{2} \Delta_{y}+\gamma_{23} \Delta_{x} \Delta_{y}^{2}\right) \\
0
\end{array}\right] .
\end{aligned}
$$

B.2. Coefficient Matrices of $\mathbf{A}_{c}$ and $\mathbf{C}_{c}$ with the Linear Critical Depth of Cut in (37) and (38). Consider

$$
\begin{aligned}
\mathbf{A}_{c} & =\left[\begin{array}{cccc}
-2 \zeta_{x} & -\omega_{x}^{2}+\delta_{z c} \alpha_{11} & 0 & \delta_{z c} \beta_{11} \\
1 & 0 & 0 & 0 \\
0 & \delta_{z c} \alpha_{21} & -2 \zeta_{y} & -\omega_{y}^{2}+\delta_{z c} \beta_{21} \\
0 & 0 & 1 & 0
\end{array}\right], \\
\mathbf{C}_{c} & =\left[\begin{array}{cccc}
0 & -\delta_{z c} \alpha_{11} & 0 & -\delta_{z c} \beta_{11} \\
0 & 0 & 0 & 0 \\
0 & -\delta_{z c} \alpha_{21} & 0 & -\delta_{z c} \beta_{21} \\
0 & 0 & 0 & 0
\end{array}\right] .
\end{aligned}
$$

$$
\mathbf{F}^{(2)}=\left[\begin{array}{c}
\delta_{z c}\left(\alpha_{12} X_{2}^{(1) 2}+\beta_{12} X_{4}^{(1) 2}+\gamma_{11} X_{2}^{(1)} X_{4}^{(1)}\right) \\
0 \\
\delta_{z c}\left(\alpha_{22} X_{2}^{(1) 2}+\beta_{22} X_{4}^{(1) 2}+\gamma_{21} X_{2}^{(1)} X_{4}^{(1)}\right) \\
0
\end{array}\right]
$$

B.3. Coefficient Matrices of $\mathbf{A}_{\gamma}$ and $\mathbf{C}_{\gamma}$ with the Perturbed Depth of Cut in (39). Consider

$$
\begin{aligned}
\mathbf{A}_{\gamma} & =\left[\begin{array}{cccc}
0 & \delta_{z \gamma} \alpha_{11} & 0 & \delta_{z \gamma} \beta_{11} \\
0 & 0 & 0 & 0 \\
0 & \delta_{z \gamma} \alpha_{21} & 0 & \delta_{z \gamma} \beta_{21} \\
0 & 0 & 1 & 0
\end{array}\right], \\
\mathbf{C}_{\gamma} & =\left[\begin{array}{cccc}
0 & -\delta_{z \gamma} \alpha_{11} & 0 & -\delta_{z \gamma} \beta_{11} \\
0 & 0 & 0 & 0 \\
0 & -\delta_{z \gamma} \alpha_{21} & 0 & -\delta_{z \gamma} \beta_{21} \\
0 & 0 & 0 & 0
\end{array}\right] .
\end{aligned}
$$

B.4. Forcing Functions of $\mathbf{F}^{(2)}$ and $\mathbf{F}^{(3)}$ in (38) and (39). Consider 


$$
\begin{aligned}
& \mathbf{F}^{(3)} \\
& =\left[\begin{array}{c}
\delta_{z c}\left(2 \alpha_{12} X_{2}^{(1)} X_{2}^{(2)}+\alpha_{13} X_{2}^{(1) 3}+2 \beta_{12} X_{4}^{(1)} X_{4}^{(2)}+\beta_{13} X_{4}^{(1) 3}+\gamma_{11}\left(X_{2}^{(1)} X_{4}^{(2)}+X_{2}^{(2)} X_{4}^{(1)}\right)+\gamma_{12} X_{2}^{(1) 2} X_{4}^{(1)}+\gamma_{13} X_{2}^{(1)} X_{4}^{(1) 2}\right) \\
0 \\
\delta_{z c}\left(2 \alpha_{22} X_{2}^{(1)} X_{2}^{(2)}+\alpha_{23} X_{2}^{(1) 3}+2 \beta_{22} X_{4}^{(1)} X_{4}^{(2)}+\beta_{23} X_{4}^{(1) 3}+\gamma_{21}\left(X_{2}^{(1)} X_{4}^{(2)}+X_{2}^{(2)} X_{4}^{(1)}\right)+\gamma_{22} X_{2}^{(1) 2} X_{4}^{(1)}+\gamma_{23} X_{2}^{(1)} X_{4}^{(1) 2}\right) \\
0
\end{array}\right]
\end{aligned}
$$

with

$$
\begin{aligned}
& X_{2}^{(k)}=x_{2}^{(k)}\left(T_{0}, T_{2}\right)-x_{2}^{(k)}\left(T_{0}-\tau, T_{2}\right), \\
& X_{4}^{(k)}=x_{4}^{(k)}\left(T_{0}, T_{2}\right)-x_{4}^{(k)}\left(T_{0}-\tau, T_{2}\right) .
\end{aligned}
$$
B.5. Coefficient Vectors of $\mathbf{Q}_{2}^{(F 2)}, \mathbf{Q}_{-2}^{(F 2)}$, and $\mathbf{Q}_{0}^{(F 2)}$ in (42). Con-
sider

$$
\begin{aligned}
\mathbf{Q}_{2}^{(F 2)}= & {\left[\begin{array}{c}
\delta_{z c}\left(\alpha_{12} \widetilde{X}_{1}^{2}+\beta_{12} \widetilde{X}_{1}^{2} \eta_{1}^{2}+\gamma_{11} \widetilde{X}_{1}^{2} \eta_{1}\right) \\
0 \\
\delta_{z c}\left(\alpha_{12} \widetilde{X}_{1}^{2}+\beta_{12} \widetilde{X}_{1}^{2} \eta_{1}^{2}+\gamma_{11} \widetilde{X}_{1}^{2} \eta_{1}\right) \\
0
\end{array}\right], } \\
\mathbf{Q}_{-2}^{(F 2)}= & {\left[\begin{array}{c}
\delta_{z c}\left(\alpha_{12} \widetilde{X}_{-1}^{2}+\beta_{12} \widetilde{X}_{-1}^{2} \eta_{2}^{2}+\gamma_{11} \widetilde{X}_{-1}^{2} \eta_{2}\right) \\
0 \\
\delta_{z c}\left(\alpha_{12} \widetilde{X}_{-1}^{2}+\beta_{12} \widetilde{X}_{-1}^{2} \eta_{2}^{2}+\gamma_{11} \widetilde{X}_{-1}^{2} \eta_{2}\right) \\
0
\end{array}\right], } \\
\mathbf{Q}_{0}^{(F 2)}= & {\left[\begin{array}{c}
\delta_{z c}\left(2 \alpha_{12} \widetilde{X}_{1}^{2} \widetilde{X}_{-1}^{2}+2 \beta_{12} \widetilde{X}_{1}^{2} \widetilde{X}_{-1}^{2} \eta_{1} \eta_{2}+\gamma_{11} \widetilde{X}_{1} \widetilde{X}_{-1}\left(\eta_{1}+\eta_{2}\right)\right) \\
0 \\
\delta_{z c}\left(2 \alpha_{22} \widetilde{X}_{1}^{2} \widetilde{X}_{-1}^{2}+2 \beta_{22} \widetilde{X}_{1}^{2} \widetilde{X}_{-1}^{2} \eta_{1} \eta_{2}+\gamma_{21} \widetilde{X}_{1} \widetilde{X}_{-1}\left(\eta_{1}+\eta_{2}\right)\right) \\
0
\end{array}\right], }
\end{aligned}
$$

where

$$
\begin{aligned}
\widetilde{X}_{1} & =1-e^{-i \omega_{c} \tau}, \\
\widetilde{X}_{-1} & =1-e^{i \omega_{c} \tau} .
\end{aligned}
$$

B.6. Shape Vector Functions of the Complex Harmonics of $\mathbf{P}_{2}^{(2)}$ $\mathbf{P}_{-2}^{(2)}$, and $\mathbf{P}_{0}^{(2)}$ in (43). Consider

B.7. Coefficient Vector of $\mathbf{Q}_{1}^{(F 3)}$ in (45). Consider

$$
\begin{aligned}
& \mathbf{Q}_{1}^{(F 3)} \\
& =\left[\begin{array}{c}
\delta_{z c}\left(\widetilde{X}_{1}^{2}\left(1+e^{i \omega_{c} \tau}\right) S_{1}^{(1)}+3 \widetilde{X}_{1}^{2} \widetilde{X}_{-1} S_{2}^{(1)}+\widetilde{X}_{1}^{3} e^{i \omega_{c} \tau} S_{3}^{(1)}\right) \\
0 \\
\delta_{z c}\left(\widetilde{X}_{1}^{2}\left(1+e^{i \omega_{c} \tau}\right) S_{1}^{(2)}+3 \widetilde{X}_{1}^{2} \widetilde{X}_{-1} S_{2}^{(2)}+\widetilde{X}_{1}^{3} e^{i \omega_{c} \tau} S_{3}^{(2)}\right) \\
0
\end{array}\right],
\end{aligned}
$$

where

$$
\begin{aligned}
S_{1}^{(1)}= & -2 P_{22}^{(2)} \alpha_{12}-2 P_{24}^{(2)} \beta_{12} \eta_{2} \\
& -\gamma_{11}\left(\eta_{2} P_{22}^{(2)}+2 P_{24}^{(2)}\right),
\end{aligned}
$$

$$
\text { for } k=-2,0,2, s_{k}=i k \omega_{c} \text {. }
$$




$$
\begin{aligned}
S_{3}^{(1)}= & \left(\gamma_{12}\left(-2 \eta_{1}-\eta_{2}\right)+\gamma_{13}\left(-2 \eta_{1} \eta_{2}-\eta_{1}^{2}\right)\right), \\
S_{1}^{(2)}= & -2 P_{22}^{(2)} \alpha_{22}-2 P_{24}^{(2)} \beta_{22} \eta_{2} \\
& -\gamma_{21}\left(\eta_{2} P_{22}^{(2)}+2 P_{24}^{(2)}\right), \\
S_{2}^{(3)}= & \alpha_{23}+\beta_{23} \eta_{1}^{2} \eta_{2}, \\
S_{3}^{(2)}= & \left(\gamma_{22}\left(-2 \eta_{1}-\eta_{2}\right)+\gamma_{23}\left(-2 \eta_{1} \eta_{2}-\eta_{1}^{2}\right)\right) .
\end{aligned}
$$

B.8. Parameter Values of $\vartheta_{r 1}, \vartheta_{i 1}, \vartheta_{r 2}$, and $\vartheta_{i 2}$ in (46). The solvability condition for (45) yields the following:

$$
\left|\begin{array}{cccc}
i \omega_{c}+2 \zeta_{x} & \omega_{x}^{2}-\delta_{z c} \alpha_{11}\left(1-e^{i \omega_{c} \tau}\right) & 0 & Q_{11}^{(3)} \\
-1 & i \omega_{c} & 0 & Q_{12}^{(3)} \\
0 & -\delta_{z c} \alpha_{21}\left(1-e^{i \omega_{c} \tau}\right) & i \omega_{c}+2 \zeta_{y} & Q_{13}^{(3)} \\
0 & 0 & -1 & Q_{14}^{(3)}
\end{array}\right|
$$

$=0$,

which can be written in the following complex form:

$$
\dot{A}_{1}=-\frac{E^{(\gamma)}}{E^{(D)}} \delta_{z \gamma} A_{1}-\frac{E^{(C)}}{E^{(D)}} A_{1}^{2} A_{2}
$$

where

$$
\begin{aligned}
& E^{(k)}= E_{1} Q_{11}^{(k)}+E_{2} Q_{12}^{(k)}+E_{3} Q_{13}^{(k)}+E_{4} Q_{14}^{(k)}, \\
& k=D, \gamma, C, \\
& E_{1}=\delta_{z c} \alpha_{21}\left(1-e^{i \omega_{c} \tau}\right), \\
& E_{2}=\left(i \omega_{c}+2 \zeta_{x}\right)\left(\omega_{x}^{2}-\delta_{z c} \alpha_{11}\left(1-e^{i \omega_{c} \tau}\right)\right), \\
& E_{3}=-\omega_{c}^{2}+i \omega_{c} 2 \zeta_{x}+\omega_{x}^{2}-\delta_{z c} \alpha_{11}\left(1-e^{i \omega_{c} \tau}\right), \\
& E_{4}=\left(\omega_{c}^{2}-2 \zeta_{x} i \omega_{c}\right)\left(i \omega_{c}+2 \zeta_{y}\right) \\
&-\left(i \omega_{c}+2 \zeta_{y}\right)\left(\omega_{x}^{2}-\delta_{z c} \alpha_{11}\left(1-e^{i \omega_{c} \tau}\right)\right),
\end{aligned}
$$

from which the real constants $\vartheta_{r 1}, \vartheta_{i 1}, \vartheta_{r 2}$, and $\vartheta_{i 2}$ can be obtained as follows:

$$
\begin{aligned}
& \vartheta_{r 1}=\operatorname{Re}\left(-\frac{E^{(\gamma)}}{E^{(D)}}\right), \\
& \vartheta_{i 1}=\operatorname{Im}\left(-\frac{E^{(\gamma)}}{E^{(D)}}\right), \\
& \vartheta_{r 2}=\operatorname{Re}\left(-\frac{E^{(C)}}{E^{(D)}}\right), \\
& \vartheta_{i 1}=\operatorname{Im}\left(-\frac{E^{(C)}}{E^{(D)}}\right) .
\end{aligned}
$$

\section{A Brief Summary of the Linear Force Model}

The elemental tangential, radial, and axial components of the cutting forces can be calculated as shown here [5]:

$$
\begin{aligned}
& \mathrm{d} F_{t}(\varphi, z)=K_{t e} \mathrm{~d} S+K_{t c} t_{n} \mathrm{~d} b, \\
& \mathrm{~d} F_{r}(\varphi, z)=K_{r e} \mathrm{~d} S+K_{r c} t_{n} \mathrm{~d} b, \\
& \mathrm{~d} F_{a}(\varphi, z)=K_{a e} \mathrm{~d} S+K_{a c} t_{n} \mathrm{~d} b,
\end{aligned}
$$

where $\left(K_{t e}, K_{r e}, K_{a e}\right)$ and $\left(K_{t c}, K_{r c}, K_{a c}\right)$ are the edge and shear force coefficients, respectively, $\mathrm{d} S$ is the differential length of the cutting edge, $t_{n}$ is the uncut chip thickness normal to the cutting edge, and $\mathrm{d} b$ is the infinitesimal length of the cutting edge normal to the radius (infinitesimal chip width), which can be expressed as

$$
\mathrm{d} b=\frac{\mathrm{d} z}{\sin (\kappa)},
$$

where $\kappa$ is the immersion angle defined by the following geometric relationship:

$$
\kappa=\arccos \frac{R_{0}-z}{R_{0}} .
$$

The static uncut chip thickness normal to the cutting edge can be obtained from the immersion angle $\kappa$, feed rate $f_{z}$, and angular position $\varphi$ (refer to Figure 1) [34-36]:

$$
t_{n, \mathrm{sta}}=f_{z} \sin (\varphi) \sin (\kappa) .
$$

The dynamic uncut chip thickness normal to the cutting edge is calculated as [1]

$$
\begin{aligned}
& t_{n, \mathrm{dyn}}=((x(t)-x(t-\tau)) \sin (\varphi) \\
& \quad+(y(t)-y(t-\tau)) \cos (\varphi)) \sin (\kappa) .
\end{aligned}
$$

Using the equations derived above, the differential local forces in the Cartesian coordinate system can be obtained as follows:

$$
\begin{aligned}
\mathrm{d} F_{x}= & -\mathrm{d} F_{r} \sin (\kappa) \sin (\varphi)-\mathrm{d} F_{t} \cos (\varphi) \\
& -\mathrm{d} F_{a} \cos (\kappa) \sin (\varphi), \\
\mathrm{d} F_{y}= & -\mathrm{d} F_{r} \sin (\kappa) \cos (\varphi)+\mathrm{d} F_{t} \sin (\varphi) \\
& -\mathrm{d} F_{a j} \cos (\kappa) \cos (\varphi), \\
\mathrm{d} F_{z}= & \mathrm{d} F_{r} \cos (\kappa)-\mathrm{d} F_{a} \sin (\kappa) .
\end{aligned}
$$

Finally, the total force acting on flute $j$ can readily be obtained by integrating these differential force components along the $z$-axis.

\section{Conflict of Interests}

The authors declare that there is no conflict of interests regarding the publication of this paper. 


\section{Acknowledgment}

This work was supported by a National Research Foundation of Korea (NRF) Grant funded by the Korean Government (MSIP) (NRF-2014R1A2A1A11049579).

\section{References}

[1] H.-Y. Feng and C.-H. Menq, "The prediction of cutting forces in the ball-end milling process-I. Model formulation and model building procedure," International Journal of Machine Tools and Manufacture, vol. 34, no. 5, pp. 697-710, 1994.

[2] A. Azeem, H.-Y. Feng, and L. Wang, "Simplified and efficient calibration of a mechanistic cutting force model for ball-end milling," International Journal of Machine Tools and Manufacture, vol. 44, no. 2-3, pp. 291-298, 2004.

[3] H.-Y. Feng and N. Su, "A mechanistic cutting force model for 3D ball-end milling," Journal of Manufacturing Science and Engineering, vol. 123, no. 1, pp. 23-29, 2001.

[4] A. Azeem and H.-Y. Feng, "Cutting force prediction for ball-end mills with non-horizontal and rotational cutting motions," The International Journal of Advanced Manufacturing Technology, vol. 67, no. 5-8, pp. 1833-1845, 2013.

[5] P. Lee and Y. Altıntaş, "Prediction of ball-end milling forces from orthogonal cutting data," International Journal of Machine Tools and Manufacture, vol. 36, no. 9, pp. 1059-1072, 1996.

[6] Y.-H. Jung, J.-S. Kim, and S.-M. Hwang, "Chip load prediction in ball-end milling," Journal of Materials Processing Technology, vol. 111, no. 1-3, pp. 250-255, 2001.

[7] Y. Sun, F. Ren, D. Guo, and Z. Jia, "Estimation and experimental validation of cutting forces in ball-end milling of sculptured surfaces," International Journal of Machine Tools and Manufacture, vol. 49, no. 15, pp. 1238-1244, 2009.

[8] Z. C. Wei, M. J. Wang, J. N. Zhu, and L. Y. Gu, "Cutting force prediction in ball end milling of sculptured surface with Z-level contouring tool path," International Journal of Machine Tools and Manufacture, vol. 51, no. 5, pp. 428-432, 2011.

[9] Z. C. Wei, M. J. Wang, Y. J. Cai, and S. F. Wang, "Prediction of cutting force in ball-end milling of sculptured surface using improved Z-map," International Journal of Advanced Manufacturing Technology, vol. 68, no. 5-8, pp. 1167-1177, 2013.

[10] S. N. Melkote and W. J. Endres, "The importance of including size effect when modeling slot milling," Journal of Manufacturing Science and Engineering, vol. 120, no. 1, pp. 68-75, 1998.

[11] G. Stepan and T. Kalmar-Nagy, "Nonlinear regenerative machine tool vibrations," in Proceedings of the 16th ASME Biennial Conference on Mechanical Vibration and Noise, DETC97/ VIB-4021, pp. 1-11, Sacramento, Calif, USA, September 1997.

[12] T. Kalmár-Nagy, G. Stépán, and F. C. Moon, "Subcritical Hopf bifurcation in the delay equation model for machine tool vibrations," Nonlinear Dynamics, vol. 26, no. 2, pp. 121-142, 2001.

[13] Z. Dombovari, R. E. Wilson, and G. Stepan, "Estimates of the bistable region in metal cutting," Proceedings of the Royal Society of London A: Mathematical, Physical and Engineering Sciences, vol. 464, no. 2100, pp. 3255-3271, 2008.

[14] Z. Dombovari and G. Stepan, "On the bistable zone of milling processes," Philosophical Transactions of the Royal Society A: Mathematical, Physical and Engineering Sciences, vol. 373, no. 2051, Article ID 20140409, 2015.
[15] Y. Altıntaş and P. Lee, "Mechanics and dynamics of ball end milling," Journal of Manufacturing Science and EngineeringTransactions of the ASME, vol. 120, no. 4, pp. 684-691, 1998.

[16] Y. Altıntaş and E. Budak, "Analytical prediction of stability lobes in milling," CIRP Annals-Manufacturing Technology, vol. 44, no. 1, pp. 357-362, 1995.

[17] F. Abrari and M. A. Elbestawi, "Closed form formulation of cutting forces for ball and flat end mills," International Journal of Machine Tools and Manufacture, vol. 37, no. 1, pp. 17-27, 1997.

[18] F. Abrari, M. A. Elbestawi, and A. D. Spence, "On the dynamics of ball end milling: modeling of cutting forces and stability analysis," International Journal of Machine Tools and Manufacture, vol. 38, no. 3, pp. 215-237, 1998.

[19] E. Ozturk and E. Budak, "Dynamics and stability of fiveaxis ball-end milling," Journal of Manufacturing Science and Engineering, vol. 132, no. 2, Article ID 021003, 13 pages, 2010.

[20] Y. Altıntaş, G. Stepan, D. Merdol, and Z. Dombovari, "Chatter stability of milling in frequency and discrete time domain," CIRP Journal of Manufacturing Science and Technology, vol. 1, no. 1, pp. 35-44, 2008.

[21] A. H. Nayfeh and D. T. Mook, Nonlinear Oscillations, John Wiley \& Sons, Hoboken, NJ, USA, 2008.

[22] Y. Altıntaş, E. Shamoto, P. Lee, and E. Budak, "Analytical prediction of stability lobes in ball end milling," Journal of Manufacturing Science and Engineering, vol. 121, no. 4, pp. 586-592, 1999.

[23] E. Budak and Y. Altıntaş, "Analytical prediction of chatter stability in milling-part I: general formulation," Journal of Dynamic Systems, Measurement and Control-Transactions of the ASME, vol. 120, no. 1, pp. 22-30, 1998.

[24] E. Budak and Y. Altıntaş, "Analytical prediction of chatter stability in milling - part II: application of the general formulation to common milling systems," Journal of Dynamic Systems, Measurement, and Control, vol. 120, no. 1, pp. 31-36, 1998.

[25] E. Ozturk and E. Budak, "Chatter stability of 5-axis milling using multi-frequency solution," in Proceedings of the $3 \mathrm{rd}$ International Conference on High Performance Cutting, HPC-P066, pp. 429-444, Dublin, Ireland, June 2008.

[26] T. Insperger and G. Stépán, Semi-Discretization for Time-Delay Systems: Stability and Engineering Applications, vol. 178 of Applied Mathematical Sciences, Springer Science \& Business Media, New York, NY, USA, 2011.

[27] Y. Altıntaş, "Analytical prediction of three dimensional chatter stability in milling," JSME International Journal, Series C: Mechanical Systems, Machine Elements and Manufacturing, vol. 44, no. 3, pp. 717-723, 2001.

[28] P. Kim and J. Seok, "Bifurcation analyses on the chatter vibrations of a turning process with state-dependent delay," Nonlinear Dynamics, vol. 69, no. 3, pp. 891-912, 2012.

[29] Maplesoft, Maple 14. 2010, Maplesoft ${ }^{\mathrm{TM}}$.

[30] G. Stépán, "Modelling nonlinear regenerative effects in metal cutting," Philosophical Transactions of the Royal Society of London A: Mathematical, Physical and Engineering Sciences, vol. 359, no. 1781, pp. 739-757, 2001.

[31] Z. Dombovari, R. E. Wilson, and G. Stepan, "Estimates of the bistable region in metal cutting," Proceedings of the Royal Society A: Mathematical, Physical and Engineering Sciences, vol. 464, no. 2100, pp. 3255-3271, 2008.

[32] L. F. Shampine and S. Thompson, "Solving DDEs in matlab," Applied Numerical Mathematics, vol. 37, no. 4, pp. 441-458, 2001. 
[33] Mathworks, Matlab Version 8.2, Mathworks Inc, 2013.

[34] E. Budak, Y. Altıntaş, and E. J. A. Armarego, "Prediction of milling force coefficients from orthogonal cutting data," Journal of Manufacturing Science and Engineering, vol. 118, no. 2, pp. 216-224, 1996.

[35] J. H. Ko and D.-W. Cho, "3D ball-end milling force model using instantaneous cutting force coefficients," Journal of Manufacturing Science and Engineering, vol. 127, no. 1, pp. 1-12, 2005.

[36] R. Salami, M. H. Sadeghi, and B. Motakef, "Feed rate optimization for 3-axis ball-end milling of sculptured surfaces," International Journal of Machine Tools and Manufacture, vol. 47, no. 5, pp. 760-767, 2007. 


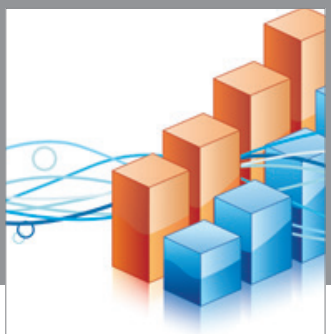

Advances in

Operations Research

vatem alat4

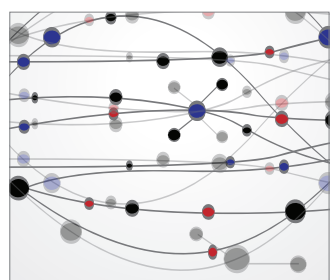

\section{The Scientific} World Journal
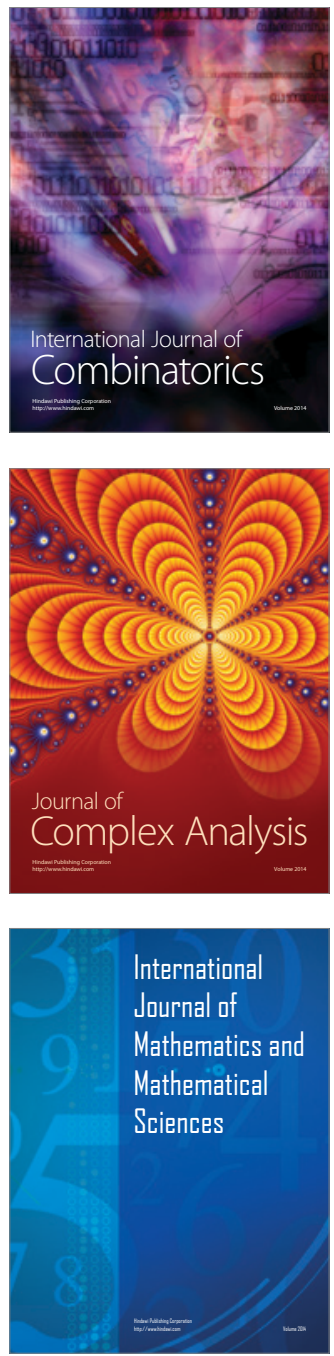
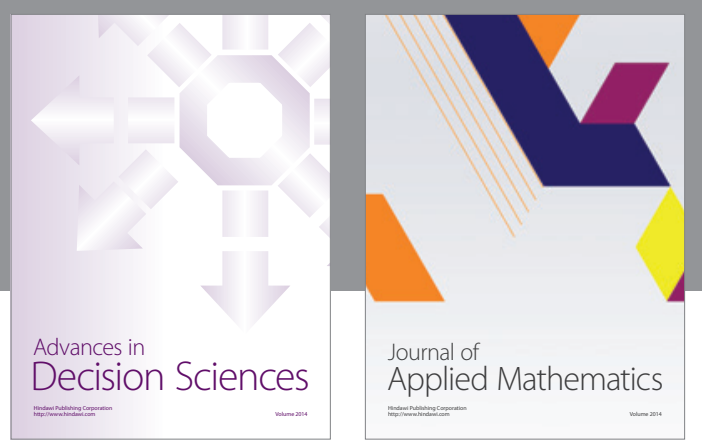

Algebra

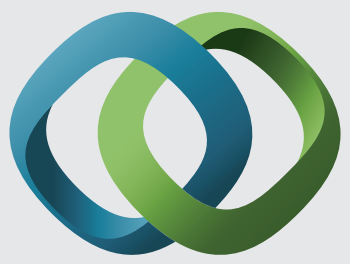

\section{Hindawi}

Submit your manuscripts at

http://www.hindawi.com
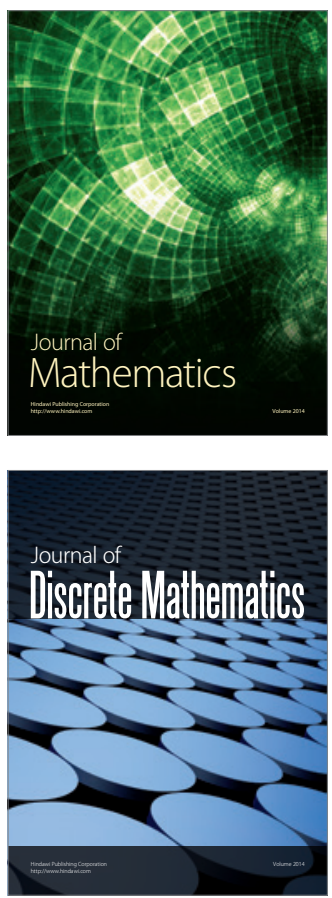

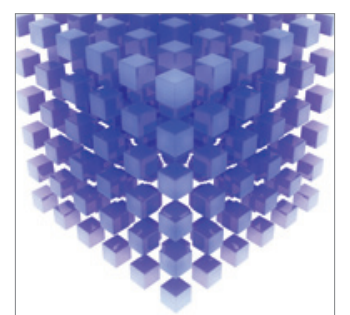

Mathematical Problems in Engineering
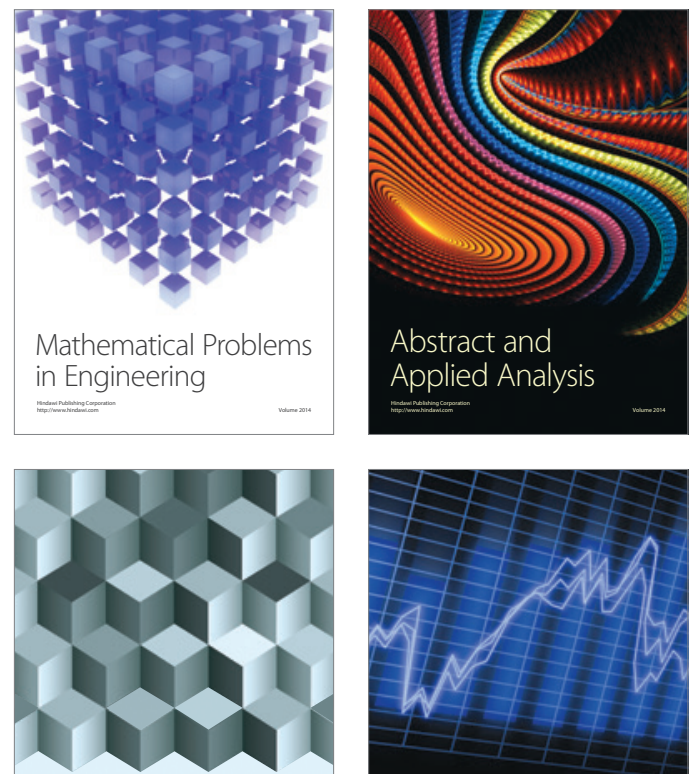

Journal of

Function Spaces

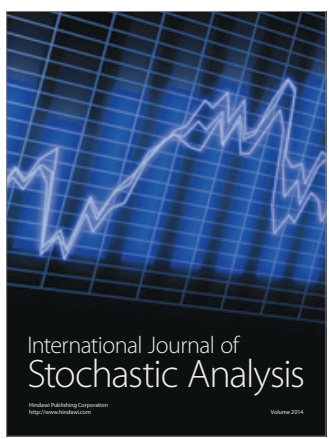

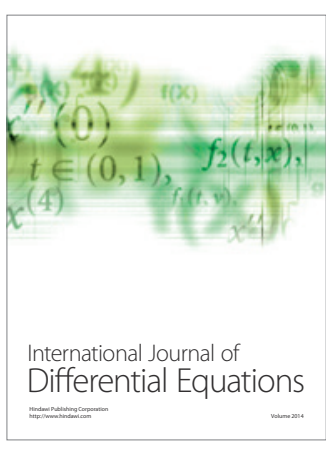
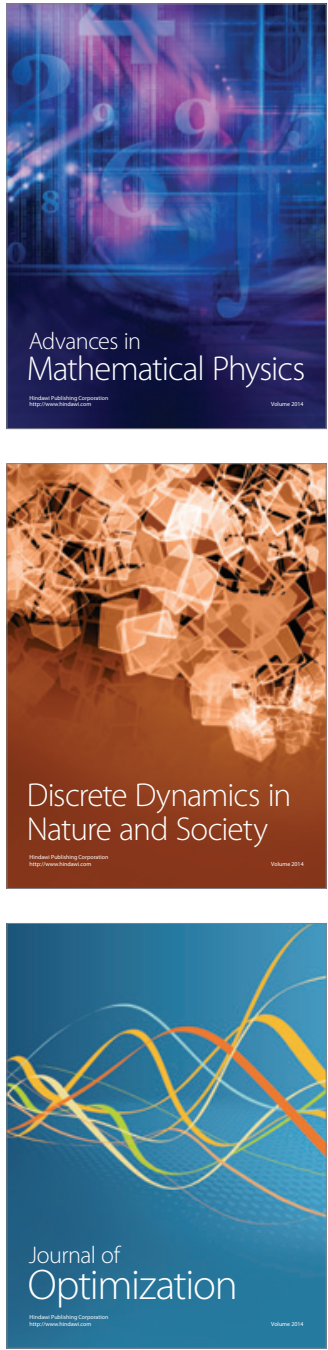